\title{
Kinematics of planetary nebulae in the outskirts of the elliptical galaxy NGC $4697^{1}$
}

\author{
R. H. Méndez ${ }^{2}$, A. M. Teodorescu², R.-P. Kudritzki² and A. Burkert ${ }^{3}$ \\ mendez@ifa.hawaii.edu
}

\begin{abstract}
We describe the implementation of slitless radial velocity measurements of extragalactic planetary nebulae (PNs) with the $8.2 \mathrm{~m}$ Subaru telescope and its Cassegrain imaging spectrograph, FOCAS. As a first application, we have extended a previous search for PNs in NGC 4697 to larger angular distances from its center. A total of $218 \mathrm{PNs}$ were detected, and their radial velocities were measured. We have added 56 new PN detections to the existing sample of 535, observed previously with the ESO VLT + FORS imaging spectrograph; 36 of these new $56 \mathrm{PNs}$ are located at angular distances larger than 230 arcsec from the center of NGC 4697. We compare the new FOCAS velocities with the earlier FORS velocities, for 158 of the 162 reobserved sources, finding good agreement. We now have kinematic information extending out to 5 effective radii from the center. The outer line-of-sight velocity dispersion is a bit lower than estimated earlier. This result is compatible with the existence of a dark matter halo plus some degree of radial anisotropy, but the dark matter halo is rather inconspicuous, and it is still unclear how massive it can be. A more detailed global dynamical study of the whole set of PN velocities will be required to decide if they permit to narrow down the range of possible dark matter distributions in NGC 4697. The new radial velocities reveal no evidence of rotation at 5 effective radii.
\end{abstract}

Subject headings: galaxies: elliptical and lenticular, cD - galaxies: individual (NGC 4697) — galaxies: kinematics and dynamics — planetary nebulae: general — techniques: radial velocities

\footnotetext{
${ }^{1}$ Based on data collected at the Subaru Telescope, which is operated by the National Astronomical Observatory of Japan.

${ }^{2}$ Institute for Astronomy, University of Hawaii, 2680 Woodlawn Drive, Honolulu, HI 96822

${ }^{3}$ Universitäts-Sternwarte München, Scheinerstr. 1, D-81679 München, Germany
} 


\section{Introduction}

Planetary nebulae (PNs) in the outskirts of elliptical galaxies offer valuable kinematic information that can be used to trace the angular momentum distribution and mass distribution in these galaxies. The classic example is the study of PNs in NGC 5128 by Hui et al. (1995), later extended by Peng et al. (2004) to 780 PNs. A dark matter halo and significant outer rotation were detected. A recent dynamical study of 340 globular clusters in NGC 5128 has shown additional evidence of a dark matter halo (Woodley et al. 2007). Another case where a dark matter halo was immediately apparent from PN kinematics is NGC 1344 (Teodorescu et al. 2005).

In a previous work (Méndez et al. 2001), hereafter Paper I, we presented photometry and slitless radial velocity information for $535 \mathrm{PNs}$ in the flattened elliptical galaxy NGC 4697. These PNs provided kinematic information up to a distance of 3 effective radii from the center of NGC 4697. The most important result in Paper I was a substantial decline of line-of-sight velocity dispersion (losvd) as a function of angular distance from the center, which can be interpreted as indicating the absence of a dark matter halo around this galaxy, if we assume an isotropic velocity distribution. However, some dark matter can be present if the velocity distribution is radially anisotropic; we explicitly warned about this alternative interpretation in Paper I, and the point has been made in much more detail by Dekel et al. (2005) and De Lorenzi et al. (2008a).

It is important to clarify which of the two interpretations, lack of dark matter or pronounced radial anisotropy, is correct. In the current context, with dark matter firmly established as one of the basic components of the universe, the existence of one or several substantial, medium-size elliptical galaxies without a dark matter halo (which is expected to have made possible its very formation) is surprising. On the other hand, if we are able to confirm radial anisotropy, then we have learned about an important constraint concerning the formation of elliptical galaxies. For example, Athanassoula (2005) has made numerical experiments in which a quick succession of disk mergers is more likely to produce significant radial anisotropy.

De Lorenzi et al. (2008a) made a detailed dynamical analysis of NGC 4697, using the PNs of Paper I and long-slit absorption line kinematic data. Their best-fitting models were radially anisotropic, and included potentials with a variety of dark matter halos. The models

with no dark matter were not consistent with the PN losvds. However, more PN velocities at larger angular distances from the galaxy's center were required to narrow down the range of acceptable dark matter models. In this paper we provide more such PN velocities.

Romanowsky et al. (2003) showed similarly declining losvds in NGC 3379, NGC 
4494 and NGC 821, claiming that their "orbit library analysis" ruled out extreme orbital anisotropies at least in the case of NGC 3379. More recent modeling (De Lorenzi et al. 2008b) indicates again that there are mass-shape-anisotropy degeneracies that need to be resolved to reach a firm conclusion. In addition, a study of two dozens of globular clusters in NGC 3379 (Pierce et al. 2006) suggests that some dark matter may be present there. A recent long-slit spectroscopic study by Forestell \& Gebhardt (2008) seems to indicate the presence of a dark matter halo around NGC 821, again in contradiction to what Romanowsky et al. (2003) concluded from the PNs in that galaxy. In summary, the case for no dark matter appears to be weakening, but it is still quite possible that less massive ellipticals have, on average, lower dark matter fractions, i.e. less easily detected dark matter halos (e.g. Napolitano et al. 2005). We seem to be in a situation that will require more careful studies of several galaxies before we can arrive to more solid conclusions.

The need to collect large PN samples means that we need to detect faint PNs. This can be better done using the largest telescopes available; so we had a strong motivation to extend the slitless radial velocity method, used in Paper I with VLT+FORS, to other large telescopes in the northern hemisphere. The FOCAS spectrograph of Subaru proved to be a good choice. As a first application of the slitless technique with FOCAS and Subaru, we have extended our search for PNs around NGC 4697 to larger angular distances. In this paper we present the resulting data and a preliminary analysis. In Section 2 we review the basic idea of slitless radial velocities and describe the Subaru + FOCAS observations in the outskirts of NGC 4697. Section 3 describes the reduction and calibration procedures. Section 4 deals with quality control of the slitless radial velocities using observations of the local PN NGC 7293. In Section 5 we give a catalog of 218 PNs detected with FOCAS, and compare the FOCAS with FORS slitless radial velocities for 158 PNs. Having verified the quality of the data, in Section 6 we discuss the line-of-sight velocity dispersion, and we analyze a plot of the escape velocity as a function of projected distance from the center of the galaxy. We adopt a distance of $10.5 \mathrm{Mpc}$ for NGC 4697, taken from Paper I. Section 7 deals with rotation in the outskirts of NGC 4697. Section 8 gives a summary of results.

\section{Radial velocity method, field selection and observations}

Since the slitless radial velocity method was described in Paper I, here we give only a brief summary of the basic ideas. Planetary nebulae can be detected in the light of [O III] $\lambda 5007$ using the traditional on-band, off-band filter technique. Having taken the on-band

image, insertion of a grism as dispersing element produces not only dispersion but also a displacement of all images. The dispersed images of PNs remain point sources, which permits 
an accurate measurement of the displacement. Calibration of the displacement as a function of wavelength and position in the CCD detector offers an efficient way of measuring radial velocities for all PNs in the field, irrespective of their number and distribution. Since no slits are used, there are no light losses, and it is not necessary to go through the complex selection and preparation procedures typical of multi-object slit (or fiber) spectroscopy. Of course the quality of the slitless velocities will depend on the seeing and will decrease for very faint sources because of the larger position uncertainty.

The project reported here involves the $8.2 \mathrm{~m}$ Subaru telescope on Mauna Kea, Hawaii, and its Cassegrain imaging spectrograph FOCAS. We wanted to extend our search for PNs to larger angular distances from the center of NGC 4697, but allowing for partial overlap with the FORS E and W fields described in Paper I, to be able to compare the radial velocities measured with both instruments. Figure 1 shows the FORS fields studied in Paper I, and the two FOCAS fields observed for the present work.

The Subaru + FOCAS observations were made on the nights of 2004 May 12/13, 13/14, and 2005 May 11/12, 12/13 and 13/14. The 2004 nights were partly clouded. The first two 2005 nights were photometric, and the last one was affected by thin cirrus. During both observing runs the seeing was stable, oscillating around 0.6 arcsec.

FOCAS has been described by Kashikawa et al. (2002). The circular field of view of FOCAS has a diameter of 6 arc minutes. This field is covered by two CCDs of $2 \mathrm{k} \times 4 \mathrm{k}$ (pixel size $15 \mu \mathrm{m}$ ) with an unexposed gap of 5 arcsec between them. The image scale is 0.1 arcsec $\mathrm{pix}^{-1}$. Two fields, E and W of NGC 4697, were observed, as shown in Figure 1.

On-band direct imaging was done through the interference filter N502, which has the following characteristics: central wavelength $5025 \AA$, peak transmission 0.68, and FWHM $60 \AA$. Off-band imaging was done through the broad-band standard visual filter. Dispersed images were obtained by exposing through filter N502 and an echelle grism with 175 grooves $\mathrm{mm}^{-1}$, operating in the 4th order. In this way the echelle grism gives a dispersion of 0.5 $\AA \mathrm{pix}^{-1}$, with good efficiency and a rather small displacement. Dispersed PN images of 0.5 arcsec have a size of 5 pixels on the CCD. This implies a radial velocity resolution of 140 $\mathrm{km} \mathrm{s}^{-1}$, i.e. the PN's internal velocity field is not resolved. In other words, PNs should always appear as single point sources. Assuming position errors of 0.4 pixel, the expected uncertainty in radial velocity is $10 \mathrm{~km} \mathrm{~s}^{-1}$.

Table 1 lists the most important CCD images obtained for this project. It includes the exposures of the FOCAS E and W NGC 4697 fields, the spectrophotometric standard G13831 (Oke 1990), and the calibration and quality control exposures to be described in next Section. Since there are two CCD images for each field (Chips 1 and 2), for brevity we have 
listed only the exposure number corresponding to Chip 1. For Chip 2, add 1 to the listed exposure number. All images were binned $2 \times 1$ in the horizontal direction (perpendicular to dispersion). This was done to increase the signal from the very faint sources we wanted to detect, but at the same time without compromising the spectral resolution. As a consequence of this binning, each point source looks like an ellipse. Unbinning is required to bring all stars back to their normal appearance.

\section{Reduction and calibration procedures}

The basic CCD reductions (bias subtraction, flat-field correction using twilight flats) were made using IRAF1 standard tasks. Then we unbinned the images, recovering the original CCD pixel numbers in the horizontal direction.

For image registration and combination we first selected, for each of the $\mathrm{E}$ and $\mathrm{W}$ fields, a pair (undispersed, dispersed) of on-band individual images, of the best possible quality, taken consecutively at the telescope, and adopted them as reference images. For field E, chip 1, the reference images were 60989 and 60991. For field W, chip 1, the reference images were 52471 and 52473. Obviously, for chip 2 the reference images were 60990, 60992, 52472 and 52474 . All the other images, including the off-band ones, were registered upon the corresponding reference image. Registration and combination were made using IRAF standard tasks. In particular the dispersed images were registered using the brightest PN images as reference point sources, avoiding in this way any dependence on ambient temperature variations (see the corresponding discussion in Section 3 of Paper I).

We still have to describe the calibration images for radial velocities. They were obtained using an engineering mask with punched holes. The mask produces an array of point sources covering the whole field when illuminated by the internal FOCAS lamps or by any extended astronomical source. There are almost 1000 calibration points, separated by about 100 pixels in $x$ and $y$. Two kinds of calibration images were obtained illuminating the engineering mask with a Th-Ar comparison lamp: (u1) undispersed, taken through filter N502; (d1) dispersed, taken through N502 and the echelle grism. In addition, there were "quality control" images, obtained illuminating the engineering mask with the local PN NGC 7293: (u2) undispersed, taken through N502; (d2) dispersed, taken through N502 and the echelle grism. Figure 2 shows examples of images $\mathrm{u} 2, \mathrm{~d} 2$ and $\mathrm{d} 1$.

\footnotetext{
${ }^{1}$ IRAF is distributed by the National Optical Astronomical Observatories, operated by the Association of Universities for Research in Astronomy, Inc., under contract to the National Science Foundation
} 
A comparison between the left and central images in Figure 2 shows the effect of inserting the grism; comparing to the position of a fixed CCD defect, easily recognizable, it is clear that all the point sources have been displaced upwards. The right image in Figure 2 shows what happens if we keep the grism in the light path and illuminate the mask with the Thorium-Argon comparison lamp; now each point source gives a Th-Ar spectrum. The positions of the Th emission lines permit to obtain the wavelength calibration for each grid point. The Th-Ar lamp is ideally suited for our purposes, because its spectrum shows several emission lines within the on-band filter transmission curve. In Paper I we needed to take the calibration images through a broader filter, because the FORS spectrographs do not have Th-Ar lamps.

Measurement of the undispersed and dispersed calibration images permits to obtain the displacement produced by the insertion of the grism as a function of wavelength and position on the CCD. The slitless radial velocity calculation proceeds in the following way: (1) identify the four calibration points closest to the undispersed position of the PN; (2) from the displacement between undispersed and dispersed PN images, calculate the redshifted wavelength of the [O III] emission at each of the four calibration points; (3) obtain the final wavelength by bilinear interpolation, weighting the four wavelength values according to the distance from each of the four calibration points to the $\mathrm{PN}$; (4) calculate the radial velocity from the final wavelength and the heliocentric correction.

\section{Radial velocity quality control using NGC 7293}

The NGC 7293 images we obtained permit to measure radial velocities in two ways: (a) slitless, using images $\mathrm{u} 2$ and $\mathrm{d} 2$ to obtain the displacement, and using the displacement as a measure of wavelength and therefore velocity, in the way we described at the end of the previous section; (b) "classical", using images d1 and d2, and treating each mask hole as a slit. Figure 3 shows a comparison of slitless vs. classical radial velocities of NGC 7293. There is good agreement, which indicates that the calibration of the displacement works very well. In addition, the average heliocentric velocity of NGC 7293 from all the grid points turns out to be approximately $-30 \mathrm{~km} \mathrm{~s}^{-1}$, in good agreement with the known systemic velocity ( $-27 \mathrm{~km} \mathrm{~s}^{-1}$, measured by Meaburn et al. 2005). Furthermore, individual grid points show a range of velocities, from -15 to $-50 \mathrm{~km} \mathrm{~s}^{-1}$. Clearly in some cases the mask holes have isolated gas parcels that are moving predominantly towards or away from us, while in most cases we see gas moving in both directions, so that the unresolved emission line profile gives us a velocity closer to the systemic velocity. From the observed range we infer a lower limit of about $20 \mathrm{~km} \mathrm{~s}^{-1}$ for the expansion velocity in [O III], again perfectly 
consistent with the $25 \mathrm{~km} \mathrm{~s}^{-1}$ measured by Meaburn et al. (2005). No grid point has given a radial velocity departing more from the systemic velocity than we should expect from the expansion velocity. From all these results we estimate that the uncertainty in FOCAS slitless velocities is of the order of $10 \mathrm{kms}^{-1}$. In the next section we will provide more evidence about the quality of the FOCAS slitless velocities.

\section{PN detection, photometry, catalog, and comparison with FORS photometry and radial velocities}

We searched for PNs using the traditional blinking method. In the same way as in Paper I, a PN candidate was found by blinking on-band vs. off-band, and confirmed by blinking undispersed vs. dispersed on-band. The source was accepted as a PN candidate if it was absent in the off-band image, and if it was a point source. A total of 218 PNs were found, 162 of which had been discovered previously with FORS. Consequently, we have added 56 new detections. Most of the new detections (36 in fact) are at angular distances larger than 230 arcsec from the center of NGC 4697.

After measuring the positions of the 218 PNs on the undispersed and dispersed images, the radial velocities were calculated as described in sections 2 and 3. We also measured the Jacoby magnitudes $m(5007)$ using the same spectrophotometric standard (G 138-31, Oke 1990) and the same procedures described in Paper I. For a definition of Jacoby magnitudes, see Jacoby (1989) or Paper I. The standard G 138-31 has $m(5007)=19.36$ (this number depends on the characteristics of the on-band filter). The faintest objects detected both in Paper I and in the present search have $m(5007) \approx 28.5$. We expect both PN searches to have similar limiting magnitudes, because the telescope sizes, instrumental and CCD efficiencies, and total exposure times, are similar.

J2000 equatorial coordinates for all PNs were calculated using a set of astrometric programs written by David Tholen and kindly provided by Fabrizio Bernardi. A first program, when given an input file with field and camera parameters, identifies all the USNO-B1 catalog stars available within the desired field and produces a list of reference stars with rough estimates of their position in the chip. The next programs make an improved centroid fitting for reference stars, producing an output file with a list of reference stars with pixel coordinates $x, y$ accurate to a few hundredths of a pixel. The last program performs the final astrometric fit, rejecting outliers and iterating, and provides the PN equatorial coordinates with uncertainties of about 0.5 arcsec.

In Table 2 we list the following for all detected PNs: identification number; $(x, y)$ coor- 
dinates, in arcsec, relative to the optical center of NGC 4697; J2000 equatorial coordinates; Jacoby magnitude, $m(5007)$; heliocentric radial velocity in $\mathrm{km} \mathrm{s}^{-1}$; and $\mathrm{E}$ and W FORS field identification numbers, if the PN is present in the FORS PN catalog (Méndez et al. 2008). FOCAS identification numbers that begin with 20,21, 22 and 23 correspond respectively to FOCAS fields $\mathrm{W}$ chip 1, W chip 2, E chip 1, and E chip 2. A few numbers are missing for a variety of reasons: object 2008's radial velocity is $281 \mathrm{~km} \mathrm{~s}^{-1}$, if the emission line is identified as [O III] $\lambda 5007$. Since this velocity is incompatible with NGC 4697, we assume that object 2008 is a background emission-line galaxy, at such a redshift that some other emission line falls into the on-band filter transmission curve (e.g. Ly $\alpha$ at $z=3.1$ ). Object 2023 was a rediscovery of 2014. Object 2049 was not convincingly identified in the grism image, and was rejected as a PN.

Since we purposely reobserved many PNs discovered previously with FORS, we can test the accuracy of equatorial coordinates, Jacoby magnitudes, and, most important, radial velocities. Figure 4 shows the differences in declination as a function of the differences in right ascension. We conclude that our coordinates are reliable to within one arcsec, which is adequate for our purposes.

Figure 5 shows the differences in magnitudes $m(5007)$ plotted as a function of the FORS $m(5007)$, which is taken from Méndez et al. (2008). There is no systematic difference, and the dispersion increases, as expected, towards fainter magnitudes. We find no need to rediscuss the photometric results and PNLF distance of 10.5 Mpc obtained in Paper I.

Figure 6 (left panel) shows FORS radial velocities as a function of FOCAS radial velocities for 158 PNs. There is satisfactory agreement. We should explain that four of the 162 PNs could not be measured in Paper I, because these PNs were too far to the right of the W FORS field (see Section 6 in Paper I).

We have generated simulations of radial velocity sets affected by random errors that follow a gaussian distribution. In Paper I we estimated errors of about 35 or $40 \mathrm{~km} \mathrm{~s}^{-1}$ for the FORS velocities. The right panel in Figure 6 shows what happens if we assume FOCAS errors of the same magnitude. The dispersion is too large. The central panel in Figure 6 was obtained assuming FOCAS errors of $20 \mathrm{~km} \mathrm{~s}^{-1}$. This is a much better representation of what we observe in the left panel, and we conclude that The FOCAS errors are not larger than $20 \mathrm{~km} \mathrm{~s}^{-1}$; in other words, the FOCAS errors are significantly smaller than the FORS errors. This result is expected. The FOCAS radial velocities are better mainly because the spectral dispersion is larger: $0.5 \AA$ per pixel versus the FORS dispersion of $1.2 \AA$ per pixel. For similar position measurement errors, the larger dispersion gives more accurate velocities. Figure 7 shows the radial velocity difference FOCAS - FORS as a function of the apparent Jacoby magnitude $m(5007)$ for 158 PNs. There is a small systematic difference of about 15 
$\mathrm{km} \mathrm{s}^{-1}$, in the sense that FORS velocities are larger, but it is well within the uncertainty attributed to FORS velocities. Note also that large differences occur only for faint PNs. This is expected, because the quality of the slitless radial velocities depends on the quality of the position measurements. For very faint sources, the positions in undispersed and dispersed images are less reliable because the sources are barely detectable and the $\mathrm{S} / \mathrm{N}$ ratio is low.

\section{Line-of-sight velocity dispersions and escape velocities}

As mentioned in the introduction, the detailed dynamical analysis by De Lorenzi et al. (2008a) showed that the best-fitting models are radially anisotropic, and require some dark matter; but it is not clear how much. What we need is more PN radial velocities at large angular distances from the center of NGC 4697. Here we present a preliminary discussion of the new FOCAS PN velocities. To illustrate how much we have improved the information about PNs distant from the center, we can mention that we have discovered a total (FORS + FOCAS) of 42 PNs at angular distances larger than 280 arcsec from the center of NGC 4697. We have FORS velocities for 10 of these outlying PNs, and we have FOCAS velocities for 41 of them. Figure 8 shows the positions of all FORS and FOCAS PNs relative to the center of light of NGC 4697 (left panel), and their radial velocities as a function of the $x$ coordinates (right panel). Our coordinate system is defined with the $x$-axis coincident with the major axis of NGC 4697, and with the origin at the center of the galaxy, measuring $x$ and $y$ in arcsec. Since the FORS data were discussed by De Lorenzi et al. (2008a), here we concentrate mostly on the new FOCAS velocities.

In a first attempt we defined six data groups: PNs with $x<-300,-300<x<-200$, $-200<x<-100,100<x<200,200<x<300$, and $300<x$. The numbers of PNs within each group were, respectively, 14, 28, 49, 47, 30 and 8 . We calculated the line-ofsight velocity dispersions (losvds), and corrected them for the small effect of measurement errors by subtracting $10 \mathrm{~km} \mathrm{~s}^{-1}$ in quadrature. Figure 9 shows the resulting losvd for each group, as a function of average angular distance from the center of NGC 4697. We also show dispersions derived from absorption-line spectra along the major axis by Binney et al. (1990), and dispersions derived from FORS PNs in Paper I. All these data are in good agreement with each other where they overlap.

De Lorenzi et al. (2008a) defined zones with elliptical boundaries for losvd calculations; see their Figure 6. Their outermost datapoint, at a radius of 300 arcsec, has a losvd of 110 $\mathrm{km} \mathrm{s}^{-1}$. We have calculated the losvd for a group involving all the 48 FOCAS PNs that lie outside an ellipse with semi-major and minor axes of 280 and 180 arcsec, respectively. The resulting losvd is $93 \pm 13 \mathrm{~km} \mathrm{~s}^{-1}$, somewhat smaller than the De Lorenzi losvd. We made a 
similar calculation for the 21 FOCAS PNs lying outside a larger ellipse, with semi-major and minor axes of 350 and 225 arcsec. The corresponding losvd is even smaller: $81 \pm 18 \mathrm{~km} \mathrm{~s}^{-1}$. These values are similar to those shown in Figure 9. We conclude, first, that our FOCAS losvds in the outskirts of NGC 4697 are not very sensitive to the shape of the regions used to define the samples; and second, that the new FOCAS data indicate a slightly smaller losvd beyond 250 arcsec than obtained from the FORS data in De Lorenzi et al. (2008a). Following their discussion, we conclude that the best fitting models are still those including a not very massive dark matter halo, but it is still difficult to narrow down the range of acceptable dark matter models, because the new FOCAS data lie somewhat closer to the models without dark matter than the earlier FORS data.

We also built a new sample by adding, to the 21 FOCAS PNs mentioned above, the six other FORS PNs that lie outside of the larger ellipse. We did not make any correction for the larger errors of those few FORS velocities. Still, the losvd for this new 27-object sample was $95 \pm 18 \mathrm{~km} \mathrm{~s}^{-1}$, again lower than the De Lorenzi et al. value.

We can also consider an argument used in the pioneering work of Hui et al. (1995) to confirm the existence of a dark matter halo around NGC 5128. When we plot PN radial velocities as a function of angular distance from the center of that galaxy, we find several PNs with velocities exceeding the local escape velocity for a Hernquist (1990) model with constant $M / L$ ratio; see their Figure 20. The argument was repeated and improved in Peng et al. (2004); see their Figure 12. Using a constant $M / L$ Hernquist model, several PNs in NGC 5128 are unbound; we would expect such stars to escape in a very short time. Instead, using a two-component Hernquist model, which includes a dark matter halo, even the most distant PNs become bound, because the escape velocity becomes much larger. This is a test we can easily apply to NGC 4697. Figure 10 shows the result. We calculated the escape velocity for a constant $M / L$ Hernquist (1990) model at a distance $r$ from the center of the mass distribution:

$$
V_{\mathrm{esc}}=\left(2 G M_{\mathrm{t}} /(r+a)\right)^{0.5}
$$

where $M_{\mathrm{t}}$ is the total mass (for our test we use two masses, $1.5 \times 10^{11} M_{\odot}$ and $9 \times 10^{10} M_{\odot}$ ), and $a$ is a scale length equal to $R_{\mathrm{e}} / 1.8153$.

The choice of galaxy mass needs to be explained. De Lorenzi et al. (2008a) found a $M / L$ ratio of about 5 in the $\mathrm{R}$ band, which is equivalent to approximately 7.5 in the $\mathrm{B}$ band (see e.g. Maraston 1998). Knowing the extinction-corrected $B_{T}=10.0$, the distance modulus 30.1 from Paper I, and the solar $B$ absolute magnitude 5.48, we obtain for NGC 4697 a blue luminosity of $1.7 \times 10^{10} L_{\odot}$, which then gives a mass of $1.3 \times 10^{11} M_{\odot}$. 
Note that the $(M / L)_{B}$ ratio of 11 determined in Paper I needs to be changed, because we have reduced the effective radius $R_{\mathrm{e}}$ of NGC 4697 after the photometric study by De Lorenzi et al. (2008a). The new value of $R_{\mathrm{e}}$ is 66 arcsec, which is equivalent to $3.36 \mathrm{kpc}$ at the galaxy's distance of $10.5 \mathrm{Mpc}$. This forces a decrease in the total mass required for a fit of the losvd with the Hernquist model. Please refer to formulas (32) and (41) in Hernquist (1990). The dispersion depends on the mass and the scale length $a$. If we change $R_{\mathrm{e}}$, which is proportional to $a$, then we need to change $M$. This is perhaps more easily appreciated in Appendix B of Hernquist (1990), where all the formulas for the dispersion express it as a function of $M / a$. Figure 9 shows, for illustrative purposes only, the result of fitting the observed losvd with a Hernquist model. Clearly the NMAGIC modeling by De Lorenzi et al. is to be preferred, but it is useful to show that the Hernquist model is not far off; the total mass of this Hernquist nodel is $1.5 \times 10^{11} M_{\odot}$, which implies $(M / L)_{B}=9$. This differs from the NMAGIC solution by less than $20 \%$ in the mass.

Let us go back to Figure 10. We have plotted two escape velocity curves, corresponding to $(M / L)_{B}=9$ and 5 . For a $(M / L)_{B}$ ratio of 9 we do not find any unbound PNs, but if we decrease $(M / L)_{B}$ to 5 , then a few PNs become unbound. It should be clear that this kind of diagram cannot prove the absence of dark matter. Since we are dealing with projected distances to the center, some PNs can in fact be located at larger distances, where the escape velocity is lower. It is also possible that some PNs have large tangential velocities, so that the total spatial velocity can exceed the escape velocity.

In summary, for the larger $M / L$ ratio, no dark matter is necessary; but, for the smaller $M / L$ ratio, the presence of several unbound $\mathrm{PNs}$ requires the presence of a dark matter halo. A curve based on the NMAGIC mass of NGC 4697 falls between the two curves we plotted, and therefore the evidence is inconclusive. We can only say that the dark matter halo around NGC 4697 is less conspicuous than the one around NGC 5128.

What is needed now is to repeat the NMAGIC modeling globally, taking into account both the old FORS and new FOCAS PN velocities. This will require considerable work by other researchers, and is outside the scope of the present paper, which is limited to presenting the new information we have obtained, and exploring its possible significance.

\section{Rotation}

In Paper I we reported that the rotation curve of NGC 4697 appeared to drop beyond about 100 arcsec from the center. We can reinvestigate the rotation at large angular distances from the center of NGC 4697 using our FOCAS PN velocities, which extend further out and 
have smaller errors than FORS velocities. Figure 11 shows histograms for PNs progressively more distant from the center. We show separate histograms for PNs with negative and positive $x$ coordinates. In the left panel there is a peak of positive relative radial velocities at negative $x$, but anyway the signal of rotation (most of the galaxy moving toward us at negative $x$, and away from us at positive $x$ ), is clear. The same sense of rotation is also present in the central panel. The right panel shows that, at $x$ coordinates larger than 300 arcsec in absolute value, the rotation is no longer evident. From Paper I and De Lorenzi et al. (2008a) we know already that there is no rotation along the minor axis (that is to say, around the major axis).

From long-slit absorption-line studies (Binney et al. 1990, De Lorenzi et al. 2008a) we know how the stars are rotating. Figure 12 shows that the PNs rotate in the same sense. We have selected PNs within 50 arcsec of the major axis, and divided them in eight groups along the major axis, with limits at $-300,-200,-100,0,100,200$ and 300 arcsec. The two extreme groups turned out to have too few PNs, and therefore we removed the $y$ limit in those two cases. In order of increasing average $x$, the numbers of PNs in each group are 14, $16,19,20,11,20,13$ and 8 . We averaged the PN radial velocities and plotted the averages as a function of the average $x$ of each group. The drop in rotation velocity at large radii is clear. It seems that NGC 4697 is hardly rotating at all at $5 R_{\mathrm{e}}$. The rotational signal must be somewhat diluted, because we were forced to include PNs with rather large $y$ coordinates; but still the behavior of rotation is obvious. Figures 11 and 12 confirm what we suspected in Paper I. In relation to this lack of outer rotation, it may be interesting to mention a recent study of the S0 galaxy NGC 1023 (Noordermeer et al. 2008), which has revealed a similar drop in the outer rotation velocity.

Sufficiently massive dark matter halos are usually expected to generate significant rotation in the outskirts, as discussed by De Lorenzi et al. (2008a). Indeed, for example NGC 5128 shows clear evidence of outer rotation (Woodley et al. 2007). That this is not the case in NGC 4697 should not be interpreted as necessarily indicating the lack of a dark matter halo. Instead, it may be a problem of how the specific angular momentum is distributed in the system, independently of its potential well and, by this, its total gravitational mass. In the same way as with the interpretation of the losvd, the interpretation of rotation will require more careful global modeling with NMAGIC or similar codes.

For the moment, the conclusion we want to extract is that there appears to exist a variety of cases concerning how much angular momentum resides in the outskirts of elliptical galaxies. PNs are a good tool that will allow to collect angular momentum information for several other galaxies. We hope that the information derived from PN radial velocities will make it possible to explore if this variety in angular momentum distribution can be traced 
to different galaxy formation mechanisms involving mergers.

\section{Summary of conclusions}

We have demonstrated how Subaru and FOCAS can be used to obtain accurate slitless radial velocities of extragalactic PNs. Working in two fields in the outskirts of NGC 4697, we discovered and provided radial velocities for 218 PNs, of which 162 had been previously observed with VLT+FORS. Where possible, we compared the FOCAS radial velocities with those previously obtained with FORS, finding good agreement. We now have PN kinematic information extending out to 5 effective radii from the center of NGC 4697 (its effective radius is 66 arcsec). In particular, we have significantly improved the kinematic information at angular distances larger than 230 arcsec from the center of NGC 4697.

We have found the following: (1) the losvd at radii between 300 and 400 arcsec from the center of NGC 4697 is below $100 \mathrm{~km} \mathrm{~s}^{-1}$, i.e. a bit lower than obtained from the much smaller FORS outer PN sample by De Lorenzi et al. (2008a). This makes it difficult to narrow down the range of acceptable dark matter models. At least we can say that the dark matter halo around NGC 4697 is not as conspicuous as the one around NGC 5128. (2) the rotational signal, which is obvious close to the center, is no longer visible at about 5 effective radii from the center. By comparison to NGC 5128, it seems that much less angular momentum resides in the outskirts of NGC 4697. We need similar kinematic information about more elliptical galaxies.

A more quantitative interpretation of these results concerning NGC 4697 will require careful global modeling of the full, enlarged, FOCAS+FORS PN data set, plus existing absorption-line kinematic data, using NMAGIC or similar codes, following procedures described by De Lorenzi et al. (2008a).

This work was supported by the National Science Foundation under Grant No. 0307489. It is a pleasure to acknowledge the help provided by the Subaru staff, in particular the support astronomers Youichi Ohyama, Takashi Hattori and Kentaro Aoki. We would like to thank Fabrizio Bernardi for his help using David Tholen's astrometric programs for the calculation of J2000 equatorial coordinates. We also acknowledge comments by R. Saglia, O. Gerhard and J. Barnes, as well as some very useful remarks by an anonymous referee. 


\section{REFERENCES}

Athanassoula, E. 2005, in AIP Conference Proceedings 804, Planetary Nebulae as Astronomical Tools, eds. R. Szczerba, G. Stasinska \& S.K. Gorny, American Institute of Physics, p. 333

Binney, J. J., Davies, R. L., \& Illingworth, G. D. 1990, ApJ, 361, 78

De Lorenzi, F., Gerhard, O., Coccato, L., et al. 2008b, astro-ph arXiv:0804.3350

De Lorenzi, F., Gerhard, O., Saglia, R.P., et al. 2008a, MNRAS, 385, 1729

Dekel, A., Stoehr, F., Mamon, G.A., et al. 2005, Nature, 437, 707

Forestell, A.D., \& Gebhardt, K. 2008, astro-ph arXiv:0803.3626

Hernquist, L. 1990, ApJ, 356, 359

Hui, X., Ford, H.C., Freeman, K.C., \& Dopita, M.A. 1995, ApJ, 449, 592

Jacoby, G.H. 1989, ApJ, 339, 39

Kashikawa, N., Aoki, K., Asai, R., et al. 2002, PASJ, 54, 819

Maraston, C. 1998, MNRAS, 300, 872

Meaburn, J., Boumis, P., López, J.A., et al. 2005, MNRAS, 360, 963

Méndez, R.H., Riffeser, A., Kudritzki, R.P., et al. 2001, ApJ, 563, 135 (Paper I)

Méndez, R.H., Teodorescu, A.M., \& Kudritzki, R.P. 2008, ApJS, 175, 522

Napolitano, N.R., Capaccioli, M., Romanowsky, A.J., et al. 2005, MNRAS, 357, 691

Noordermeer, E., Merrifield, M.R., Coccato, L., et al. 2008, MNRAS, 384, 943

Oke, J.B. 1990, AJ, 99, 1621

Peng, E.W., Ford, H.C., \& Freeman, K.C. 2004, ApJ, 602, 685

Pierce, M., Beasley, M.A., Forbes, D.A., et al., 2006, MNRAS, 366, 1253

Romanowsky, A.J., Douglas, N.G., Arnaboldi, M., et al. 2003, Science, 301, 1696

Teodorescu, A.M., Méndez, R.H., Saglia, R.P., et al. 2005, ApJ, 635, 290

Woodley, K.A., Harris, W.E., Beasley, M.A., et al. 2007, AJ, 134, 494 
This preprint was prepared with the AAS LATEX macros v5.2. 
Table 1. Observations and calibrations

\begin{tabular}{|c|c|c|c|c|}
\hline FOCAS Field & Configuration & FOCAS number & $\exp (\mathrm{s})$ & Air mass ${ }^{\mathrm{a}}$ \\
\hline NGC $7293+$ mask & on-band & 52385 & 200 & 1.88 \\
\hline NGC 7293 + mask & on + grism & 52387 & 300 & 1.83 \\
\hline Th-Ar + mask & on + grism & 52391 & 10 & 1.77 \\
\hline NGC $4697 \mathrm{~W}$ & off-band & 52445 & 120 & 1.17 \\
\hline NGC $4697 \mathrm{~W}$ & on-band & 52447 & 1200 & 1.15 \\
\hline NGC $4697 \mathrm{~W}$ & on + grism & 52449 & 1800 & 1.12 \\
\hline Th-Ar + mask & on + grism & 52453 & 10 & 1.11 \\
\hline Th-Ar + mask & on-band & 52455 & 4 & 1.11 \\
\hline NGC $4697 \mathrm{~W}$ & off-band & 52457 & 120 & 1.11 \\
\hline NGC $4697 \mathrm{~W}$ & on-band & 52459 & 1200 & 1.11 \\
\hline NGC $4697 \mathrm{~W}$ & on + grism & 52461 & 1800 & 1.12 \\
\hline Th-Ar + mask & on + grism & 52465 & 10 & 1.14 \\
\hline Th-Ar + mask & on-band & 52467 & 4 & 1.14 \\
\hline NGC $4697 \mathrm{~W}$ & off-band & 52469 & 120 & 1.15 \\
\hline NGC $4697 \mathrm{~W}$ & on-band & 52471 & 1200 & 1.17 \\
\hline NGC $4697 \mathrm{~W}$ & on + grism & 52473 & 1800 & 1.23 \\
\hline NGC $4697 \mathrm{~W}$ & off-band & 52481 & 120 & 1.31 \\
\hline NGC $4697 \mathrm{~W}$ & on-band & 52483 & 1200 & 1.36 \\
\hline NGC $4697 \mathrm{~W}$ & on + grism & 52485 & 1800 & 1.70 \\
\hline NGC $4697 \mathrm{E}$ & off-band & 60793 & 120 & 1.24 \\
\hline NGC $4697 \mathrm{E}$ & on-band & 60795 & 1200 & 1.21 \\
\hline NGC $4697 \mathrm{E}$ & on + grism & 60797 & 1800 & 1.16 \\
\hline NGC $4697 \mathrm{E}$ & off-band & 60803 & 120 & 1.13 \\
\hline NGC $4697 \mathrm{E}$ & on-band & 60805 & 1200 & 1.12 \\
\hline NGC $4697 \mathrm{E}$ & on + grism & 60807 & 1800 & 1.11 \\
\hline NGC $4697 \mathrm{E}$ & off-band & 60813 & 120 & 1.12 \\
\hline NGC $4697 \mathrm{E}$ & on-band & 60815 & 1200 & 1.13 \\
\hline NGC $4697 \mathrm{E}$ & on + grism & 60817 & 1800 & 1.16 \\
\hline NGC $4697 \mathrm{E}$ & off-band & 60823 & 120 & 1.22 \\
\hline NGC $4697 \mathrm{E}$ & on-band & 60825 & 1200 & 1.26 \\
\hline NGC $4697 \mathrm{E}$ & on + grism & 60827 & 1800 & 1.35 \\
\hline
\end{tabular}


Table 1-Continued

\begin{tabular}{lrlrc}
\hline \hline FOCAS Field & Configuration & FOCAS number & exp (s) & Air mass \\
\hline NGC 4697 E & on + grism & 60833 & 2000 & 1.59 \\
G 138-31 & on-band & 60843 & 60 & 1.02 \\
G 138-31 & on-band & 60845 & 120 & 1.02 \\
G 138-31 & on-band & 60847 & 60 & 1.02 \\
G 138-31 & on-band & 60849 & 60 & 1.02 \\
G 138-31 & on-band & 60851 & 120 & 1.02 \\
G 138-31 & on-band & 60879 & 60 & 1.34 \\
G 138-31 & on-band & 60881 & 120 & 1.36 \\
G 138-31 & on-band & 60883 & 60 & 1.38 \\
G 138-31 & on-band & 60885 & 120 & 1.40 \\
NGC 4697 W & off-band & 60967 & 120 & 1.24 \\
NGC 4697 W & on-band & 60969 & 1200 & 1.21 \\
NGC 4697 W & on + grism & 60971 & 1800 & 1.16 \\
NGC 4697 W & off-band & 60977 & 120 & 1.12 \\
NGC 4697 W & on-band & 60979 & 1200 & 1.12 \\
NGC 4697 W & on + grism & 60981 & 1800 & 1.11 \\
NGC 4697 E & off-band & 60987 & 120 & 1.12 \\
NGC 4697 E & on-band & 60989 & 1200 & 1.13 \\
NGC 4697 E & on + grism & 60991 & 1800 & 1.16 \\
Th-Ar + mask & on + grism & 60993 & 10 & 1.20 \\
Th-Ar + mask & on-band & 60995 & 4 & 1.20 \\
NGC 4697 E & off-band & 60997 & 120 & 1.21 \\
NGC 4697 E & on-band & 60999 & 1200 & 1.24 \\
NGC 4697 E & on + grism & 61001 & 1800 & 1.35 \\
NGC 4697 W & off-band & 61011 & 120 & 1.55 \\
NGC 4697 W & on-band & 61013 & 1200 & 1.65 \\
NGC 4697 W & on + grism & 61015 & 120 & 1.96 \\
G 138-31 & on-band & 61017 & 1.02 \\
G 138-31 & on-band & 61019 & 1.02 \\
G 138-31 & on-band & 61021 & 1.03 \\
G 138-31 & on-band & 61023 & &
\end{tabular}


Table 1-Continued

\begin{tabular}{lrlrl}
\hline \hline FOCAS Field & Configuration & FOCAS number & exp (s) & Air mass \\
\hline NGC 4697 E & off-band & 61081 & 140 & 1.24 \\
NGC 4697 E & on-band & 61083 & 1400 & 1.20 \\
NGC 4697 E & on + grism & 61085 & 2200 & 1.15 \\
NGC 4697 E & off-band & 61091 & 140 & 1.12 \\
NGC 4697 E & on-band & 61093 & 1400 & 1.11 \\
NGC 4697 E & on + grism & 61095 & 2200 & 1.12 \\
NGC 4697 W & off-band & 61101 & 140 & 1.13 \\
NGC 4697 W & on-band & 61103 & 1400 & 1.15 \\
NGC 4697 W & on + grism & 61105 & 2200 & 1.22 \\
NGC 4697 W & off-band & 61111 & 140 & 1.30 \\
NGC 4697 W & on-band & 61113 & 1400 & 1.36 \\
NGC 4697 W & on + grism & 61115 & 2200 & 1.54 \\
NGC 4697 W & on + grism & 61119 & 2200 & 1.93 \\
\hline
\end{tabular}

athe air masses correspond to the middle of each exposure 
Table 2. Planetary nebulae in NGC 4697 detected with FOCAS ${ }^{a}$

\begin{tabular}{|c|c|c|c|c|c|c|c|c|c|c|c|c|}
\hline Id, F & $x, \mathrm{G}$ & $y, \mathrm{G}$ & & $\alpha$ & $(2000)$ & & $\delta$ & $(2000)$ & $m(5007)$ & Helioc. RV & Id, E & Id, W \\
\hline 2001 & 376 & -2 & 12 & 48 & 13.13 & -5 & 50 & 43.3 & 27.02 & 1256 & -1 & -1 \\
\hline 2002 & 378 & -128 & 12 & 48 & 16.59 & -5 & 52 & 38.1 & 27.01 & 1240 & -1 & -1 \\
\hline 2003 & 386 & -129 & 12 & 48 & 16.12 & -5 & 52 & 42.5 & 26.98 & 1282 & -1 & -1 \\
\hline 2004 & 287 & -191 & 12 & 48 & 23.89 & -5 & 52 & 56.8 & 26.67 & 1314 & -1 & -1 \\
\hline 2005 & 312 & -16 & 12 & 48 & 17.45 & -5 & 50 & 28.9 & 27.84 & 1134 & -1 & -1 \\
\hline 2006 & 338 & -142 & 12 & 48 & 19.37 & -5 & 52 & 34.1 & 28.33 & 1362 & -1 & -1 \\
\hline 2007 & 286 & -13 & 12 & 48 & 18.91 & -5 & 50 & 15.3 & 25.90 & 1367 & -1 & 1746 \\
\hline 2009 & 281 & -69 & 12 & 48 & 20.79 & -5 & 51 & 3.5 & 26.49 & 1178 & -1 & 1742 \\
\hline 2010 & 278 & -94 & 12 & 48 & 21.71 & -5 & 51 & 25.4 & 27.51 & 1400 & -1 & -1 \\
\hline 2011 & 281 & -136 & 12 & 48 & 22.70 & -5 & 52 & 4.1 & 27.60 & 1251 & -1 & -1 \\
\hline 2012 & 260 & -155 & 12 & 48 & 24.48 & -5 & 52 & 12.9 & 27.90 & 1346 & -1 & -1 \\
\hline 2013 & 250 & -130 & 12 & 48 & 24.40 & -5 & 51 & 45.7 & 28.06 & 1326 & -1 & -1 \\
\hline 2014 & 236 & -80 & 12 & 48 & 23.82 & -5 & 50 & 54.6 & 26.47 & 1218 & -1 & 1734 \\
\hline 2015 & 260 & -61 & 12 & 48 & 21.84 & -5 & 50 & 48.0 & 26.95 & 1370 & -1 & 1743 \\
\hline 2016 & 269 & -34 & 12 & 48 & 20.52 & -5 & 50 & 27.2 & 26.93 & 1312 & -1 & 1745 \\
\hline 2017 & 271 & -44 & 12 & 48 & 20.71 & -5 & 50 & 36.5 & -1 & 1450 & -1 & 1744 \\
\hline 2018 & 239 & -8 & 12 & 48 & 21.59 & -5 & 49 & 50.9 & 27.43 & 1208 & -1 & -1 \\
\hline 2019 & 236 & -5 & 12 & 48 & 21.71 & -5 & 49 & 46.5 & 27.97 & 1309 & -1 & -1 \\
\hline 2020 & 224 & -38 & 12 & 48 & 23.38 & -5 & 50 & 11.6 & 27.09 & 1472 & -1 & 1736 \\
\hline 2021 & 216 & -28 & 12 & 48 & 23.62 & -5 & 49 & 59.0 & 25.97 & 1332 & -1 & 1737 \\
\hline 2022 & 211 & -45 & 12 & 48 & 24.37 & -5 & 50 & 12.8 & 27.19 & 1280 & -1 & 1735 \\
\hline 2024 & 235 & -92 & 12 & 48 & 24.26 & -5 & 51 & 4.5 & 27.16 & 1368 & -1 & 1733 \\
\hline 2025 & 220 & -137 & 12 & 48 & 26.43 & -5 & 51 & 40.1 & 26.03 & 1265 & -1 & 1732 \\
\hline 2026 & 196 & -150 & 12 & 48 & 28.23 & -5 & 51 & 41.1 & 27.51 & 1312 & -1 & -1 \\
\hline 2027 & 191 & -80 & 12 & 48 & 26.55 & -5 & 50 & 35.9 & 27.23 & 1135 & -1 & 1722 \\
\hline 2028 & 202 & -45 & 12 & 48 & 24.90 & -5 & 50 & 9.0 & 26.05 & 1315 & -1 & 1723 \\
\hline 2029 & 187 & -16 & 12 & 48 & 25.01 & -5 & 49 & 36.3 & 27.57 & 1399 & -1 & -1 \\
\hline 2030 & 184 & -51 & 12 & 48 & 26.20 & -5 & 50 & 6.1 & 27.10 & 1322 & -1 & -1 \\
\hline 2031 & 165 & -120 & 12 & 48 & 29.26 & -5 & 51 & 0.8 & 26.56 & 1286 & -1 & 1700 \\
\hline 2032 & 130 & -27 & 12 & 48 & 28.79 & -5 & 49 & 21.4 & 26.15 & 1430 & -1 & 1668 \\
\hline 2033 & 153 & -21 & 12 & 48 & 27.18 & -5 & 49 & 25.8 & 25.96 & 1234 & -1 & 1707 \\
\hline 2034 & 141 & -65 & 12 & 48 & 29.19 & -5 & 50 & 1.0 & 26.16 & 1097 & -1 & 1703 \\
\hline 2035 & 126 & -78 & 12 & 48 & 30.46 & -5 & 50 & 6.5 & 25.64 & 1243 & -1 & 1661 \\
\hline 2036 & 108 & -38 & 12 & 48 & 30.41 & -5 & 49 & 22.8 & 26.51 & 1193 & -1 & 1663 \\
\hline 2037 & 198 & -13 & 12 & 48 & 24.25 & -5 & 49 & 38.3 & 27.44 & 1517 & -1 & -1 \\
\hline 2038 & 74 & -25 & 12 & 48 & 32.14 & -5 & 48 & 55.9 & 25.72 & 1348 & -1 & 1627 \\
\hline 2039 & 108 & -10 & 12 & 48 & 29.62 & -5 & 48 & 57.6 & 26.29 & 1365 & -1 & 1666 \\
\hline 2040 & 92 & -27 & 12 & 48 & 31.10 & -5 & 49 & 5.3 & 26.85 & 1400 & -1 & 1664 \\
\hline 2041 & 126 & -21 & 12 & 48 & 28.84 & -5 & 49 & 15.1 & 26.63 & 1464 & -1 & 1667 \\
\hline 2042 & 85 & -51 & 12 & 48 & 32.21 & -5 & 49 & 24.5 & 26.20 & 953 & -1 & 1623 \\
\hline 2043 & 120 & -54 & 12 & 48 & 30.15 & -5 & 49 & 41.7 & 27.67 & 1449 & -1 & -1 \\
\hline 2044 & 104 & -54 & 12 & 48 & 31.09 & -5 & 49 & 35.0 & 27.08 & 1425 & -1 & 1662 \\
\hline 2045 & 120 & -103 & 12 & 48 & 31.52 & -5 & 50 & 26.9 & 27.30 & 1101 & -1 & 1660 \\
\hline 2046 & 147 & -84 & 12 & 48 & 29.34 & -5 & 50 & 21.2 & 26.78 & 1312 & -1 & 1701 \\
\hline 2047 & 133 & -78 & 12 & 48 & 30.01 & -5 & 50 & 9.1 & 26.85 & 1222 & -1 & 1702 \\
\hline 2048 & 156 & -72 & 12 & 48 & 28.48 & -5 & 50 & 13.5 & 27.67 & 1245 & -1 & -1 \\
\hline 2050 & 69 & -13 & 12 & 48 & 32.10 & -5 & 48 & 43.1 & -1 & 1407 & -1 & 1628 \\
\hline 2051 & 81 & -14 & 12 & 48 & 31.38 & -5 & 48 & 49.7 & 26.77 & 1388 & -1 & 1630 \\
\hline 2101 & 273 & 112 & 12 & 48 & 16.16 & -5 & 48 & 15.8 & 26.93 & 1261 & -1 & -1 \\
\hline 2102 & 270 & 102 & 12 & 48 & 16.62 & -5 & 48 & 23.9 & 27.53 & 1207 & -1 & -1 \\
\hline 2103 & & & & & & & & & 27.12 & 1540 & -1 & \\
\hline
\end{tabular}


Table 2-Continued

\begin{tabular}{|c|c|c|c|c|c|c|c|c|c|c|c|c|}
\hline Id, F & $x, \mathrm{G}$ & $y, \mathrm{G}$ & & $\alpha$ & $(2000)$ & & $\delta$ & (2000) & $m(5007)$ & Helioc. RV & Id, E & Id, W \\
\hline 2104 & 329 & 89 & 12 & 48 & 13.42 & -5 & 49 & 0.2 & 27.61 & 1375 & -1 & -1 \\
\hline 2105 & 313 & 60 & 12 & 48 & 15.18 & -5 & 49 & 20.5 & 26.30 & 1270 & -1 & -1 \\
\hline 2106 & 356 & 162 & 12 & 48 & 9.72 & -5 & 48 & 6.1 & 27.15 & 1276 & -1 & -1 \\
\hline 2107 & 281 & 164 & 12 & 48 & 14.18 & -5 & 47 & 32.2 & 28.16 & 1335 & -1 & -1 \\
\hline 2108 & 278 & 30 & 12 & 48 & 18.18 & -5 & 49 & 33.1 & 28.45 & 1329 & -1 & -1 \\
\hline 2109 & 241 & 66 & 12 & 48 & 19.41 & -5 & 48 & 44.2 & 26.92 & 1380 & -1 & 1738 \\
\hline 2110 & 225 & 69 & 12 & 48 & 20.30 & -5 & 48 & 34.7 & 27.67 & 1339 & -1 & 1739 \\
\hline 2111 & 211 & 79 & 12 & 48 & 20.87 & -5 & 48 & 19.9 & -1 & 1185 & -1 & 1740 \\
\hline 2112 & 199 & 67 & 12 & 48 & 21.94 & -5 & 48 & 25.4 & 26.23 & 1359 & -1 & 1728 \\
\hline 2113 & 207 & 36 & 12 & 48 & 22.31 & -5 & 48 & 57.3 & 26.08 & 1290 & -1 & 1727 \\
\hline 2114 & 207 & 26 & 12 & 48 & 22.63 & -5 & 49 & 6.2 & 27.40 & 1205 & -1 & 1725 \\
\hline 2115 & 194 & 31 & 12 & 48 & 23.24 & -5 & 48 & 55.9 & 27.65 & 1403 & -1 & 1726 \\
\hline 2116 & 203 & 15 & 12 & 48 & 23.14 & -5 & 49 & 14.5 & 27.44 & 1125 & -1 & 1724 \\
\hline 2117 & 186 & 45 & 12 & 48 & 23.35 & -5 & 48 & 40.5 & 27.68 & 1316 & -1 & -1 \\
\hline 2118 & 157 & 37 & 12 & 48 & 25.32 & -5 & 48 & 35.7 & 27.04 & 1349 & -1 & 1714 \\
\hline 2119 & 165 & 41 & 12 & 48 & 24.69 & -5 & 48 & 34.9 & 26.48 & 1204 & -1 & 1715 \\
\hline 2120 & 175 & 108 & 12 & 48 & 22.20 & -5 & 47 & 38.6 & 27.30 & 1511 & -1 & 1729 \\
\hline 2121 & 168 & 85 & 12 & 48 & 23.29 & -5 & 47 & 56.0 & 27.27 & 1339 & -1 & 1717 \\
\hline 2122 & 159 & 66 & 12 & 48 & 24.39 & -5 & 48 & 9.3 & 27.88 & 1437 & -1 & 1716 \\
\hline 2123 & 125 & 72 & 12 & 48 & 26.25 & -5 & 47 & 49.7 & 26.81 & 1401 & -1 & 1686 \\
\hline 2124 & 173 & 70 & 12 & 48 & 23.38 & -5 & 48 & 11.8 & 27.99 & 1323 & -1 & -1 \\
\hline 2125 & 114 & 116 & 12 & 48 & 25.67 & -5 & 47 & 5.6 & 26.39 & 1357 & -1 & 1692 \\
\hline 2126 & 110 & 86 & 12 & 48 & 26.80 & -5 & 47 & 30.9 & 27.68 & 1076 & -1 & 1688 \\
\hline 2127 & 121 & 85 & 12 & 48 & 26.16 & -5 & 47 & 36.5 & 28.00 & 1554 & -1 & 1689 \\
\hline 2128 & 97 & 96 & 12 & 48 & 27.27 & -5 & 47 & 16.3 & 26.80 & 1268 & -1 & 1691 \\
\hline 2129 & 99 & 82 & 12 & 48 & 27.58 & -5 & 47 & 29.7 & 26.48 & 1286 & -1 & 1687 \\
\hline 2130 & 100 & 75 & 12 & 48 & 27.73 & -5 & 47 & 36.4 & 27.05 & 1290 & -1 & 1685 \\
\hline 2131 & 94 & 70 & 12 & 48 & 28.23 & -5 & 47 & 38.2 & 26.63 & 1415 & -1 & 1684 \\
\hline 2132 & 149 & 142 & 12 & 48 & 22.85 & -5 & 46 & 56.8 & 27.51 & 1301 & -1 & 1718 \\
\hline 2133 & 152 & 138 & 12 & 48 & 22.75 & -5 & 47 & 1.2 & 27.57 & 1471 & -1 & -1 \\
\hline 2134 & 194 & 133 & 12 & 48 & 20.33 & -5 & 47 & 24.0 & 27.62 & 1265 & -1 & 1730 \\
\hline 2135 & 138 & 42 & 12 & 48 & 26.32 & -5 & 48 & 22.3 & 26.31 & 1064 & -1 & 1713 \\
\hline 2136 & 136 & 33 & 12 & 48 & 26.70 & -5 & 48 & 29.5 & 26.28 & 1175 & -1 & 1711 \\
\hline 2137 & 133 & 34 & 12 & 48 & 26.86 & -5 & 48 & 27.6 & 26.44 & 1063 & -1 & 1712 \\
\hline 2138 & 122 & 31 & 12 & 48 & 27.63 & -5 & 48 & 25.5 & 27.08 & 1323 & -1 & 1677 \\
\hline 2139 & 117 & 59 & 12 & 48 & 27.13 & -5 & 47 & 58.8 & 25.63 & 1095 & -1 & 1683 \\
\hline 2140 & 109 & 59 & 12 & 48 & 27.60 & -5 & 47 & 55.6 & 26.59 & 1240 & -1 & 1682 \\
\hline 2141 & 135 & 14 & 12 & 48 & 27.31 & -5 & 48 & 46.8 & 27.33 & 1321 & -1 & -1 \\
\hline 2142 & 135 & 2 & 12 & 48 & 27.67 & -5 & 48 & 58.0 & 27.00 & 1462 & -1 & 1710 \\
\hline 2143 & 85 & 61 & 12 & 48 & 28.98 & -5 & 47 & 42.9 & 27.03 & 1410 & -1 & -1 \\
\hline 2144 & 95 & 53 & 12 & 48 & 28.62 & -5 & 47 & 54.5 & 26.51 & 1087 & -1 & 1681 \\
\hline 2145 & 99 & 42 & 12 & 48 & 28.67 & -5 & 48 & 6.1 & 26.69 & 1315 & -1 & 1679 \\
\hline 2146 & 95 & 42 & 12 & 48 & 28.95 & -5 & 48 & 5.0 & 26.71 & 1351 & -1 & 1678 \\
\hline 2147 & 98 & 50 & 12 & 48 & 28.55 & -5 & 47 & 58.4 & 27.55 & 1287 & -1 & 1680 \\
\hline 2148 & 87 & 35 & 12 & 48 & 29.64 & -5 & 48 & 7.7 & 27.22 & 1222 & -1 & 1644 \\
\hline 2149 & 112 & 2 & 12 & 48 & 29.02 & -5 & 48 & 48.2 & 26.08 & 1303 & -1 & 1671 \\
\hline 2150 & 109 & 8 & 12 & 48 & 29.04 & -5 & 48 & 41.4 & 26.70 & 1180 & -1 & 1673 \\
\hline 2151 & 104 & 16 & 12 & 48 & 29.15 & -5 & 48 & 31.3 & 26.53 & 1377 & -1 & 1676 \\
\hline 2152 & 78 & 30 & 12 & 48 & 30.33 & -5 & 48 & 7.8 & 26.61 & 1160 & -1 & 1643 \\
\hline 2153 & 85 & 27 & 12 & 48 & 29.97 & -5 & 48 & 13.7 & 27.20 & 1170 & -1 & 1642 \\
\hline 2154 & 86 & 19 & 12 & 48 & 30.11 & -5 & 48 & 21.2 & 27.28 & 1060 & -1 & 1641 \\
\hline
\end{tabular}


Table 2-Continued

\begin{tabular}{|c|c|c|c|c|c|c|c|c|c|c|c|c|}
\hline $\mathrm{Id}, \mathrm{F}$ & $x, \mathrm{G}$ & $y, \mathrm{G}$ & & $\alpha$ & $(2000)$ & & $\delta$ & $(2000)$ & $m(5007)$ & Helioc. RV & $\mathrm{Id}, \mathrm{E}$ & $\mathrm{Id}, \mathrm{W}$ \\
\hline 2155 & 74 & 3 & 12 & 48 & 31.32 & -5 & 48 & 30.8 & 25.87 & 1414 & -1 & 1636 \\
\hline 2201 & -265 & -109 & 12 & 48 & 55.10 & -5 & 47 & 48.8 & 27.77 & 1107 & 27 & -1 \\
\hline 2202 & -393 & -128 & 12 & 49 & 3.37 & -5 & 47 & 12.2 & 27.07 & 1297 & -1 & -1 \\
\hline 2203 & -340 & -157 & 12 & 49 & 0.98 & -5 & 48 & 0.7 & 26.23 & 1257 & -1 & -1 \\
\hline 2204 & -349 & -51 & 12 & 48 & 58.55 & -5 & 46 & 21.6 & 25.70 & 1289 & -1 & -1 \\
\hline 2205 & -342 & -46 & 12 & 48 & 57.98 & -5 & 46 & 19.6 & 27.24 & 1349 & -1 & -1 \\
\hline 2206 & -331 & -57 & 12 & 48 & 57.60 & -5 & 46 & 34.6 & 26.64 & 1244 & -1 & -1 \\
\hline 2207 & -318 & -54 & 12 & 48 & 56.76 & -5 & 46 & 37.0 & 26.74 & 1173 & -1 & -1 \\
\hline 2208 & -258 & -38 & 12 & 48 & 52.63 & -5 & 46 & 47.8 & 26.55 & 1220 & 65 & -1 \\
\hline 2209 & -251 & -53 & 12 & 48 & 52.66 & -5 & 47 & 4.0 & 26.25 & 1153 & 66 & -1 \\
\hline 2210 & -244 & -52 & 12 & 48 & 52.16 & -5 & 47 & 6.2 & 26.88 & 1189 & 67 & -1 \\
\hline 2211 & $\begin{array}{l}-244 \\
-242\end{array}$ & -47 & 12 & $\begin{array}{l}40 \\
48\end{array}$ & 51.96 & -5 & 47 & $\begin{array}{l}0.2 \\
2.8\end{array}$ & $\begin{array}{l}27.00 \\
27.78\end{array}$ & 1094 & 68 & -1 \\
\hline 2212 & -228 & -26 & 12 & 48 & 50.48 & -5 & 46 & 49.4 & 27.77 & 1314 & -1 & -1 \\
\hline 2213 & -338 & -16 & 12 & 48 & 56.88 & -5 & 45 & 54.5 & 26.12 & 1261 & -1 & -1 \\
\hline 2214 & -303 & -28 & 12 & 48 & 55.06 & -5 & 46 & 19.5 & 26.72 & 1310 & 114 & -1 \\
\hline 2215 & -221 & -113 & 12 & 48 & 52.56 & -5 & 48 & 11.3 & 28.09 & 1245 & -1 & -1 \\
\hline 2216 & -261 & -131 & 12 & 48 & 55.45 & -5 & 48 & 10.9 & 27.55 & 1167 & 11 & -1 \\
\hline 2217 & -255 & -167 & 12 & 48 & 56.13 & -5 & 48 & 45.6 & 26.73 & 1085 & 2 & -1 \\
\hline 2218 & -294 & -179 & 12 & 48 & 58.82 & -5 & 48 & 40.5 & 26.65 & 1363 & 1 & -1 \\
\hline 2219 & -215 & -157 & 12 & 48 & 53.42 & -5 & 48 & 53.4 & 27.60 & 1289 & 3 & -1 \\
\hline 2220 & -217 & -33 & 12 & $\begin{array}{l}40 \\
48\end{array}$ & 50.00 & -5 & 47 & $\begin{array}{r}0.4 \\
0.7\end{array}$ & 26.78 & 1189 & 69 & -1 \\
\hline 2221 & -209 & -34 & 12 & 48 & 49.53 & -5 & 47 & 5.0 & -1 & 1224 & 70 & -1 \\
\hline 2222 & -201 & -13 & 12 & $\begin{array}{l}40 \\
48\end{array}$ & $\begin{array}{l}48.49\end{array}$ & -5 & 46 & $\begin{array}{r}0.0 \\
49.1\end{array}$ & 27.06 & 1356 & 118 & -1 \\
\hline 2223 & -178 & -30 & 12 & 48 & 47.59 & -5 & 47 & 14.1 & 27.03 & 1388 & 121 & -1 \\
\hline 2224 & -172 & -18 & 12 & 48 & 46.88 & -5 & 47 & 6.3 & 27.12 & 1297 & 120 & -1 \\
\hline 2225 & -143 & -9 & 12 & 48 & 44.83 & -5 & 47 & 10.4 & 27.43 & 1051 & 128 & -1 \\
\hline 2226 & -150 & -38 & 12 & 48 & 46.12 & -5 & 47 & 33.8 & 27.45 & 1124 & 73 & -1 \\
\hline 2227 & -124 & -11 & 12 & 48 & 43.76 & -5 & 47 & 20.3 & 27.17 & 1245 & 129 & -1 \\
\hline 2228 & -107 & -14 & 12 & 48 & 42.80 & -5 & 47 & 29.4 & 25.93 & 1495 & 130 & -1 \\
\hline 2229 & -95 & $\begin{array}{l}-29 \\
-29\end{array}$ & 12 & $\begin{array}{l}48 \\
48\end{array}$ & 42.46 & -5 & 47 & 48.5 & 26.35 & $\begin{array}{l}1409 \\
\end{array}$ & 142 & -1 \\
\hline 2230 & -83 & -23 & 12 & 48 & 41.59 & -5 & 47 & 47.8 & 26.51 & 1074 & 140 & -1 \\
\hline 2231 & $\begin{array}{l}-80 \\
-86\end{array}$ & -23 & 12 & $\begin{array}{l}40 \\
48\end{array}$ & $\begin{array}{l}41.77\end{array}$ & -5 & 47 & 46.9 & -1 & 1190 & $\begin{array}{l}140 \\
139\end{array}$ & -1 \\
\hline 2232 & -85 & -25 & 12 & 48 & 41.77 & -5 & 47 & 49.3 & 27.36 & 1486 & 141 & -1 \\
\hline $\begin{array}{l}2232 \\
2233\end{array}$ & $\begin{array}{l}-80 \\
-89\end{array}$ & -11 & $\begin{array}{l}12 \\
12\end{array}$ & $\begin{array}{l}40 \\
48\end{array}$ & 41.65 & $\begin{array}{l}-0 \\
-5\end{array}$ & $\begin{array}{l}41 \\
47\end{array}$ & $\begin{array}{l}49.3 \\
35.0\end{array}$ & 27.50 & $\begin{array}{r}1400 \\
980\end{array}$ & $\begin{array}{l}141 \\
137\end{array}$ & $\begin{array}{l}-1 \\
-1\end{array}$ \\
\hline 2234 & -79 & -14 & 12 & 48 & 41.09 & -5 & 47 & 41.8 & 26.73 & 1474 & 138 & -1 \\
\hline 2235 & -76 & -47 & 12 & 48 & 41.87 & -5 & 48 & 12.8 & 26.56 & 1015 & 82 & -1 \\
\hline 2236 & -79 & -43 & 12 & 48 & 41.94 & -5 & 48 & 7.7 & $\begin{array}{l}27.31 \\
\end{array}$ & 1115 & 81 & -1 \\
\hline 2237 & -88 & -58 & 12 & 48 & 42.90 & -5 & 48 & 17.3 & 26.89 & 1164 & 83 & -1 \\
\hline 2238 & -99 & -67 & 12 & 48 & 43.83 & -5 & 48 & 21.1 & 26.54 & 1249 & 84 & -1 \\
\hline 2239 & -118 & -69 & 12 & 48 & 45.03 & -5 & 48 & 15.4 & 26.83 & 1304 & 78 & -1 \\
\hline 2240 & -144 & -80 & 12 & $\begin{array}{l}40 \\
48\end{array}$ & $\begin{array}{l}46.03 \\
46.93\end{array}$ & -5 & 48 & $\begin{array}{l}10.4 \\
13.8\end{array}$ & $\begin{array}{l}20.03 \\
25.82\end{array}$ & 1165 & 36 & -1 \\
\hline 2241 & -132 & -107 & 12 & 48 & 46.92 & -5 & 48 & 43.6 & 26.21 & 1016 & 41 & -1 \\
\hline 2242 & -126 & -106 & 12 & $\begin{array}{l}40 \\
48\end{array}$ & $\begin{array}{l}46.59\end{array}$ & -5 & $\begin{array}{l}48 \\
48\end{array}$ & 45.0 & $\begin{array}{l}20.21 \\
26.96\end{array}$ & 1333 & $\begin{array}{l}41 \\
42\end{array}$ & -1 \\
\hline 2243 & -110 & -113 & 12 & 48 & 45.77 & -5 & 48 & 57.9 & 26.50 & 1250 & 15 & -1 \\
\hline 2244 & -137 & -87 & 12 & 48 & 46.71 & -5 & 48 & 23.3 & 27.48 & 1170 & 37 & -1 \\
\hline 2245 & -113 & -85 & 12 & 48 & 45.19 & -5 & 48 & 32.1 & 27.26 & 1019 & 40 & -1 \\
\hline 2246 & -120 & -82 & 12 & 48 & 45.49 & -5 & 48 & 26.4 & 27.63 & 1068 & 39 & -1 \\
\hline 2247 & -139 & -71 & 12 & $\begin{array}{l}40 \\
48\end{array}$ & $\begin{array}{l}46.49 \\
46.37\end{array}$ & -5 & $\begin{array}{l}48 \\
48\end{array}$ & $\begin{array}{r}0.4 \\
8.4\end{array}$ & 27.98 & $\begin{array}{l}1150 \\
1150\end{array}$ & -1 & -1 \\
\hline 2248 & -155 & -95 & 12 & 48 & 47.99 & -5 & 48 & 23.1 & 26.99 & 1246 & 35 & -1 \\
\hline 2249 & -157 & -89 & 12 & $\begin{array}{l}40 \\
48\end{array}$ & $\begin{array}{l}47.96 \\
\end{array}$ & -5 & 48 & 17.0 & $\begin{array}{l}20.97 .51 \\
20\end{array}$ & $\begin{array}{l}1280 \\
1283\end{array}$ & 34 & -1 \\
\hline 2250 & -166 & -106 & 12 & 48 & 49.02 & -5 & 48 & 28.6 & 26.51 & 1159 & 31 & -1 \\
\hline
\end{tabular}


Table 2-Continued

\begin{tabular}{|c|c|c|c|c|c|c|c|c|c|c|c|c|}
\hline Id, F & $x, \mathrm{G}$ & $y, \mathrm{G}$ & & $\alpha$ & $(2000)$ & & $\delta$ & $(2000)$ & $m(5007)$ & Helioc. RV & Id, E & Id, W \\
\hline 2251 & -169 & -109 & 12 & 48 & 49.25 & -5 & 48 & 29.5 & 27.52 & 1076 & 30 & -1 \\
\hline 2252 & -162 & -108 & 12 & 48 & 48.80 & -5 & 48 & 32.2 & 27.52 & 1307 & 32 & -1 \\
\hline 2253 & -172 & -133 & 12 & 48 & 50.11 & -5 & 48 & 50.2 & 27.06 & 1343 & 12 & -1 \\
\hline 2254 & -133 & -123 & 12 & 48 & 47.48 & -5 & 48 & 57.3 & -1 & 1241 & 13 & -1 \\
\hline 2255 & -198 & -81 & 12 & 48 & 50.21 & -5 & 47 & 51.8 & 26.60 & 1351 & 28 & -1 \\
\hline 2256 & -175 & -101 & 12 & 48 & 49.38 & -5 & 48 & 20.0 & 27.40 & 1214 & 29 & -1 \\
\hline 2257 & -179 & -64 & 12 & 48 & 48.58 & -5 & 47 & 44.9 & 27.98 & 1139 & 71 & -1 \\
\hline 2258 & -111 & -43 & 12 & 48 & 43.89 & -5 & 47 & 54.8 & 27.47 & 1176 & 76 & -1 \\
\hline 2259 & -103 & -38 & 12 & 48 & 43.24 & -5 & 47 & 53.0 & 27.34 & 1096 & 79 & -1 \\
\hline 2260 & -66 & -6 & 12 & 48 & 40.07 & -5 & 47 & 39.9 & -1 & 1140 & 136 & 1024 \\
\hline 2261 & -59 & -15 & 12 & 48 & 39.92 & -5 & 47 & 50.5 & 26.61 & 1207 & 162 & 1043 \\
\hline 2262 & $\begin{array}{l}-59 \\
-61\end{array}$ & -14 & 12 & $\begin{array}{l}40 \\
48\end{array}$ & 40.00 & -5 & 47 & 49.3 & -1 & 1123 & $\begin{array}{l}162 \\
161\end{array}$ & $\begin{array}{l}1042 \\
1042\end{array}$ \\
\hline 2301 & -88 & 71 & 12 & 48 & 39.25 & -5 & 46 & 20.6 & 26.61 & 1319 & 300 & -1 \\
\hline 2302 & -89 & 50 & 12 & 48 & 39.92 & -5 & 46 & 39.0 & 26.73 & 1218 & 301 & -1 \\
\hline 2303 & -92 & 45 & 12 & 48 & 40.22 & -5 & 46 & 43.3 & 27.45 & 1148 & 220 & -1 \\
\hline 2304 & -105 & 31 & 12 & 48 & 41.39 & -5 & 46 & 49.7 & 26.78 & 1402 & 219 & -1 \\
\hline 2305 & -86 & 29 & 12 & 48 & 40.34 & -5 & 47 & 0.1 & 26.95 & 1332 & 222 & -1 \\
\hline 2306 & -94 & 20 & 12 & 48 & 41.09 & -5 & 47 & 4.7 & 27.05 & 1341 & 221 & -1 \\
\hline 2307 & -74 & 24 & 12 & 48 & 39.72 & -5 & 47 & 9.5 & 26.55 & 973 & 226 & -1 \\
\hline 2308 & -76 & 25 & 12 & 48 & 39.79 & -5 & 47 & 7.6 & 27.24 & 1220 & 225 & -1 \\
\hline 2309 & -70 & 32 & 12 & $\begin{array}{l}40 \\
48\end{array}$ & $\begin{array}{l}39.22 \\
39\end{array}$ & -5 & 47 & 4.0 & 27.15 & 1254 & 228 & -1 \\
\hline 2310 & -63 & 34 & 12 & 48 & 38.78 & -5 & 47 & 5.1 & 26.55 & 1069 & 229 & 1050 \\
\hline 2311 & -117 & $\begin{array}{r}54 \\
129\end{array}$ & 12 & $\begin{array}{l}40 \\
48\end{array}$ & 39.34 & -5 & 45 & $\begin{array}{l}0.1 \\
16.5\end{array}$ & $\begin{array}{l}26.058 \\
26.6\end{array}$ & 1157 & 362 & -1 \\
\hline 2312 & -129 & 127 & 12 & 48 & 40.17 & -5 & 45 & 13.1 & 26.27 & 1324 & 343 & -1 \\
\hline 2313 & -137 & 119 & 12 & 48 & 40.85 & -5 & 45 & 16.3 & 27.30 & 1389 & 342 & -1 \\
\hline 2314 & -113 & 96 & 12 & 48 & 40.07 & -5 & 45 & 47.4 & 27.15 & 1011 & 346 & -1 \\
\hline 2315 & -112 & 64 & 12 & 48 & 40.87 & -5 & 46 & 16.8 & 26.78 & 1124 & 298 & -1 \\
\hline 2316 & -125 & 28 & 12 & 48 & 42.74 & -5 & 46 & 44.5 & 26.38 & 1161 & 217 & -1 \\
\hline 2317 & -130 & 26 & 12 & 48 & 43.04 & -5 & 46 & 44.3 & -1 & 1320 & 216 & -1 \\
\hline 2318 & -124 & 6 & 12 & 48 & $\begin{array}{l}43.26 \\
\end{array}$ & -5 & 47 & 4.3 & 26.88 & 1035 & 126 & -1 \\
\hline 2319 & -156 & 19 & 12 & 48 & 44.83 & -5 & 46 & 39.8 & 27.38 & 1375 & -1 & -1 \\
\hline 2320 & -172 & 7 & 12 & 48 & 46.13 & -5 & 46 & 43.4 & 26.03 & 1313 & $\begin{array}{l}-1 \\
119\end{array}$ & -1 \\
\hline 2321 & -168 & 63 & 12 & 48 & 44.32 & -5 & 45 & 54.5 & 26.54 & 1464 & 295 & -1 \\
\hline $\begin{array}{l}2321 \\
2322\end{array}$ & $\begin{array}{l}-100 \\
-149\end{array}$ & 80 & $\begin{array}{l}12 \\
12\end{array}$ & $\begin{array}{l}40 \\
48\end{array}$ & $\begin{array}{l}4.52 \\
42.68\end{array}$ & $\begin{array}{l}-0 \\
-5\end{array}$ & $\begin{array}{l}40 \\
45\end{array}$ & $\begin{array}{l}44.5 \\
46.5\end{array}$ & $\begin{array}{l}20.04 \\
27.47\end{array}$ & $\begin{array}{l}1404 \\
1211\end{array}$ & $\begin{array}{l}290 \\
296\end{array}$ & $\begin{array}{l}-1 \\
-1\end{array}$ \\
\hline 2323 & -146 & 102 & 12 & 48 & 41.90 & -5 & 45 & 28.3 & 28.22 & 1336 & 340 & -1 \\
\hline 2324 & -156 & 99 & 12 & 48 & 42.55 & -5 & 45 & 26.4 & 27.44 & 1279 & 339 & -1 \\
\hline 2325 & -151 & 93 & 12 & 48 & 42.41 & -5 & 45 & 34.0 & 27.87 & 1327 & 341 & -1 \\
\hline 2326 & -166 & 125 & 12 & 48 & 42.47 & -5 & 44 & 58.7 & 27.79 & 1349 & 337 & -1 \\
\hline 2327 & -181 & 19 & 12 & 48 & 46.37 & -5 & 46 & 28.7 & 26.68 & 1030 & -1 & -1 \\
\hline 2328 & -183 & 13 & 12 & 48 & 46.65 & -5 & 46 & 33.3 & 27.71 & 1193 & -1 & -1 \\
\hline $\begin{array}{l}2320 \\
2329\end{array}$ & -188 & 27 & 12 & $\begin{array}{l}40 \\
48\end{array}$ & $\begin{array}{l}46.055 \\
4\end{array}$ & -5 & 46 & 18.9 & 26.42 & 1331 & $\begin{array}{l}-1 \\
-1\end{array}$ & -1 \\
\hline 2330 & -188 & 26 & 12 & 48 & 46.57 & -5 & 46 & 19.5 & 26.30 & 1223 & -1 & -1 \\
\hline 2331 & $\begin{array}{l}-100 \\
-189\end{array}$ & 31 & 12 & $\begin{array}{l}40 \\
48\end{array}$ & $\begin{array}{l}46.53 \\
46.53\end{array}$ & -5 & 46 & 14.7 & $\begin{array}{l}20.50 \\
27.69\end{array}$ & 1251 & $\begin{array}{l}-1 \\
-1\end{array}$ & -1 \\
\hline 2332 & -201 & 17 & 12 & 48 & 47.64 & -5 & 46 & 21.6 & 26.90 & 1037 & 214 & -1 \\
\hline $\begin{array}{l}2332 \\
2333\end{array}$ & $\begin{array}{l}-201 \\
-206\end{array}$ & $\begin{array}{l}17 \\
19\end{array}$ & $\begin{array}{l}12 \\
12\end{array}$ & $\begin{array}{l}48 \\
48\end{array}$ & $\begin{array}{l}47.04 \\
47.86\end{array}$ & $\begin{array}{l}-5 \\
-5\end{array}$ & $\begin{array}{l}40 \\
46\end{array}$ & $\begin{array}{l}18.0 \\
18.4\end{array}$ & $\begin{array}{l}26.80 \\
26.85\end{array}$ & $\begin{array}{l}1031 \\
1275\end{array}$ & $\begin{array}{l}214 \\
213\end{array}$ & $\begin{array}{l}-1 \\
-1\end{array}$ \\
\hline 2334 & -216 & 12 & 12 & 48 & 48.70 & -5 & 46 & 20.2 & 26.84 & 1019 & 212 & -1 \\
\hline 2335 & -223 & 6 & 12 & 48 & 49.27 & -5 & 46 & 22.5 & 26.64 & 1316 & 116 & -1 \\
\hline 2336 & -204 & $\begin{array}{r}70 \\
77\end{array}$ & 12 & $\begin{array}{l}40 \\
48\end{array}$ & 46.11 & -5 & 45 & 25.9 & $\begin{array}{l}27.046 \\
27.66\end{array}$ & 1148 & $\begin{array}{l}290 \\
293\end{array}$ & -1 \\
\hline 2337 & -252 & 16 & 12 & 48 & 50.74 & -5 & 46 & 1.8 & 25.87 & 1225 & 211 & -1 \\
\hline 2338 & -247 & 5 & 12 & $\begin{array}{l}40 \\
48\end{array}$ & 50.78 & -5 & 46 & $\begin{array}{l}1.0 \\
12.9\end{array}$ & $\begin{array}{l}27.39 \\
27.3\end{array}$ & 1273 & 115 & -1 \\
\hline 2339 & -232 & 20 & 12 & 48 & 49.44 & -5 & 46 & 6.1 & 27.08 & 1285 & -1 & -1 \\
\hline
\end{tabular}


Table 2-Continued

\begin{tabular}{|c|c|c|c|c|c|c|c|c|c|c|c|c|}
\hline Id, F & $x, \mathrm{G}$ & $y, \mathrm{G}$ & & $\alpha$ & (2000) & & $\delta$ & $(2000)$ & $m(5007)$ & Helioc. RV & Id, E & Id, W \\
\hline 2340 & -272 & 161 & 12 & 48 & 47.88 & -5 & 43 & 41.5 & 27.30 & 1239 & 357 & -1 \\
\hline 2341 & -272 & 39 & 12 & 48 & 51.31 & -5 & 45 & 32.3 & 27.70 & 1267 & -1 & -1 \\
\hline 2342 & -277 & 35 & 12 & 48 & 51.73 & -5 & 45 & 33.5 & 27.74 & 1158 & -1 & -1 \\
\hline 2343 & -283 & 46 & 12 & 48 & 51.76 & -5 & 45 & 21.5 & -1 & 1267 & 210 & -1 \\
\hline 2344 & -290 & 111 & 12 & 48 & 50.37 & -5 & 44 & 19.5 & 26.97 & 1110 & 336 & -1 \\
\hline 2345 & -294 & 107 & 12 & 48 & 50.75 & -5 & 44 & 21.5 & 26.55 & 1101 & 335 & -1 \\
\hline 2346 & -338 & 143 & 12 & 48 & 52.37 & -5 & 43 & 29.6 & 27.21 & 1120 & -1 & -1 \\
\hline 2347 & -327 & 19 & 12 & 48 & 55.21 & -5 & 45 & 26.9 & 26.05 & 1425 & -1 & -1 \\
\hline 2348 & -361 & 81 & 12 & 48 & 55.54 & -5 & 44 & 16.6 & 26.62 & 1360 & -1 & -1 \\
\hline 2349 & -373 & 96 & 12 & 48 & 55.84 & -5 & 43 & 57.7 & 26.93 & 1238 & -1 & -1 \\
\hline 2350 & -403 & 101 & 12 & 48 & 57.51 & -5 & 43 & 40.8 & 26.49 & 1105 & -1 & -1 \\
\hline 2351 & -409 & 95 & 12 & 48 & 58.08 & -5 & 43 & 43.5 & 26.77 & 1229 & -1 & -1 \\
\hline 2352 & -83 & 17 & 12 & 48 & 40.48 & -5 & 47 & 11.6 & 27.39 & 1286 & 224 & -1 \\
\hline 2353 & -72 & 19 & 12 & 48 & 39.74 & -5 & 47 & 14.3 & 27.61 & 1165 & 227 & -1 \\
\hline
\end{tabular}

${ }^{\text {a }}$ The $x, \mathrm{G}$ and $y, \mathrm{G}$ pixel coordinates are given in arcsec and have their origin at the center of light of NGC 4697 The $x, \mathrm{G}$ coordinate is defined along the major axis of NGC 4697. The units of Right Ascension are hours, minutes and seconds; the units of Declination are degrees, arc minutes, and arc seconds. In col. (6) a value of -1 for $m(5007)$ indicates that no measurement was attempted, because of CCD defects or a complex background. Heliocentric radial velocitic are given in $\mathrm{km} \mathrm{s}^{-1}$. In the last two columns, a value of -1 indicates that the object either does not belong or was not 


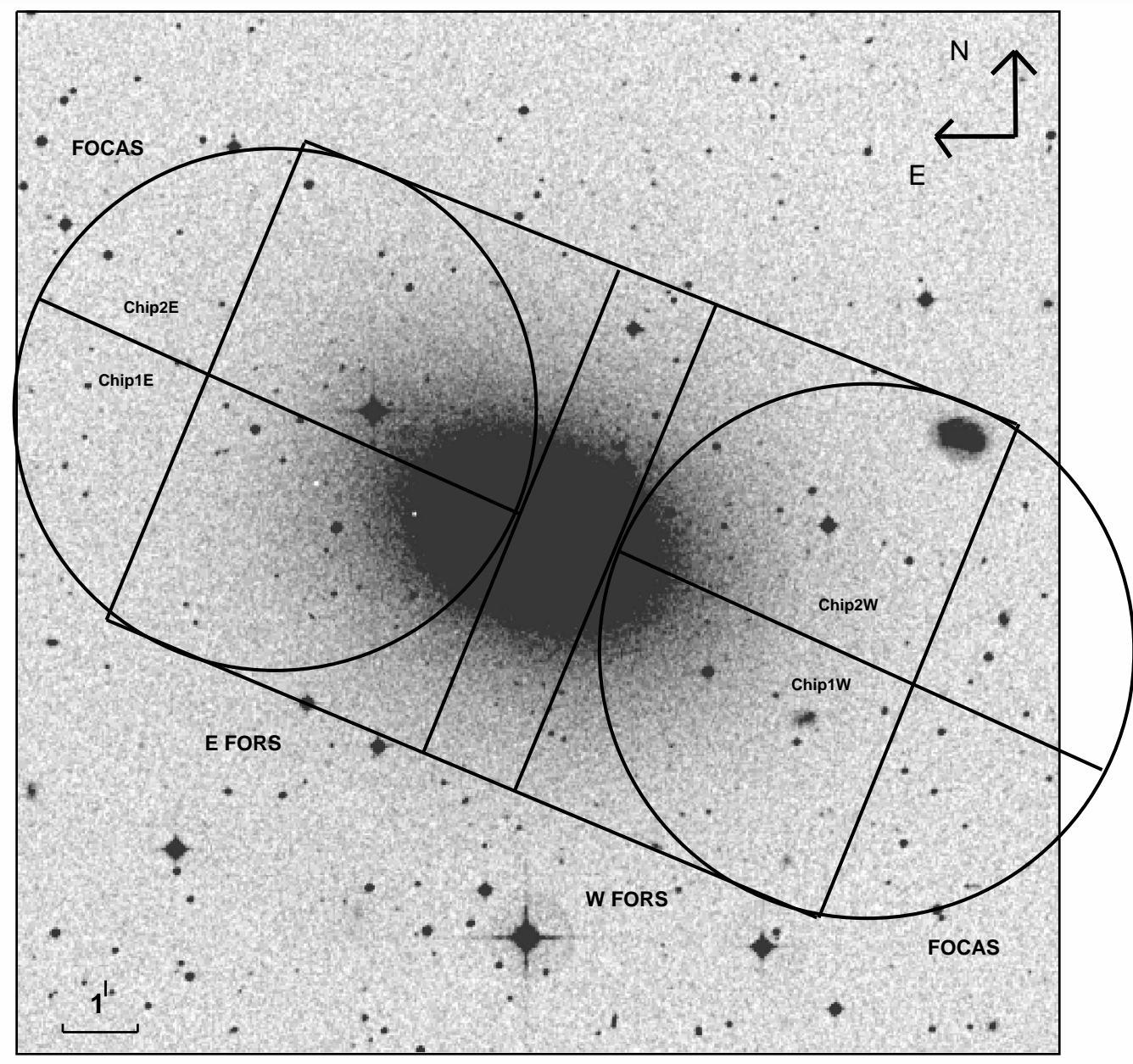

Fig. 1.- FOCAS (circular) and FORS (rectangular) fields observed in NGC 4697. Paper I was based on observations of the FORS fields. The line splitting each circular FOCAS field indicates the position of the narrow gap ( 5 arcsec) separating chip 1 from chip 2. 

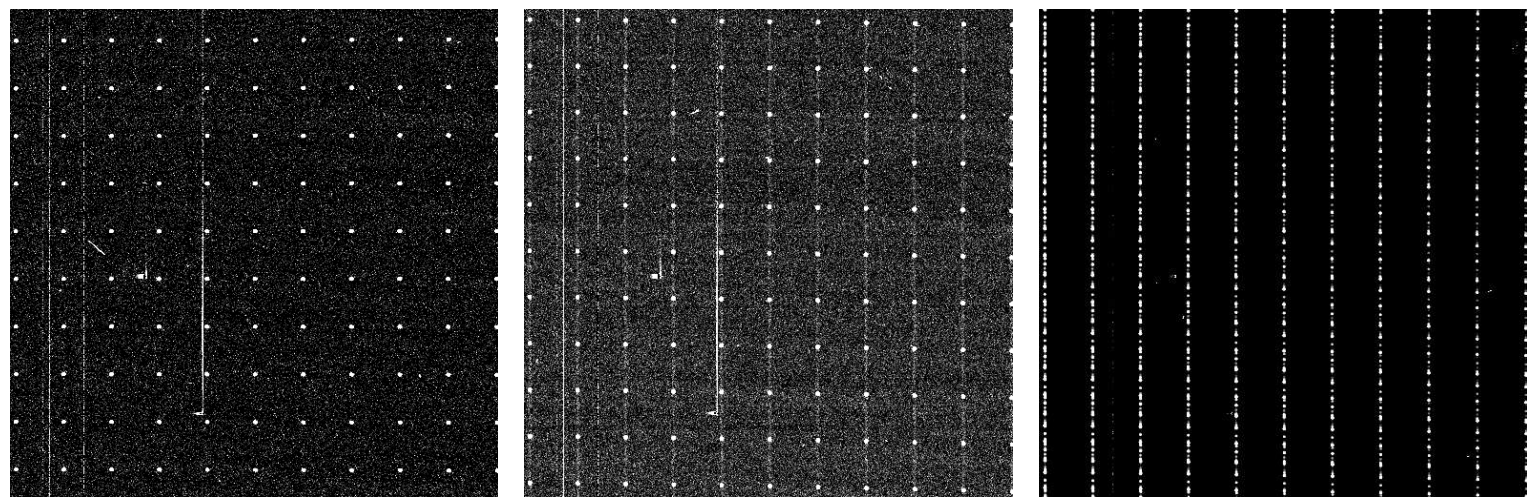

Fig. 2.- Calibration and quality control images obtained with FOCAS. From left to right, we show (u2) part of the engineering mask illuminated by NGC 7293, inserting the on-band filter; (d2) the same, adding the grism; what we see is [O III] $\lambda 5007$ at each position (the only emission line passed by the on-band filter), plus a very faint nebular continuum; and (d1) the result of illuminating the mask with the Th-Ar comparison lamp, inserting filter N502 and grism. The direction of dispersion is vertical.
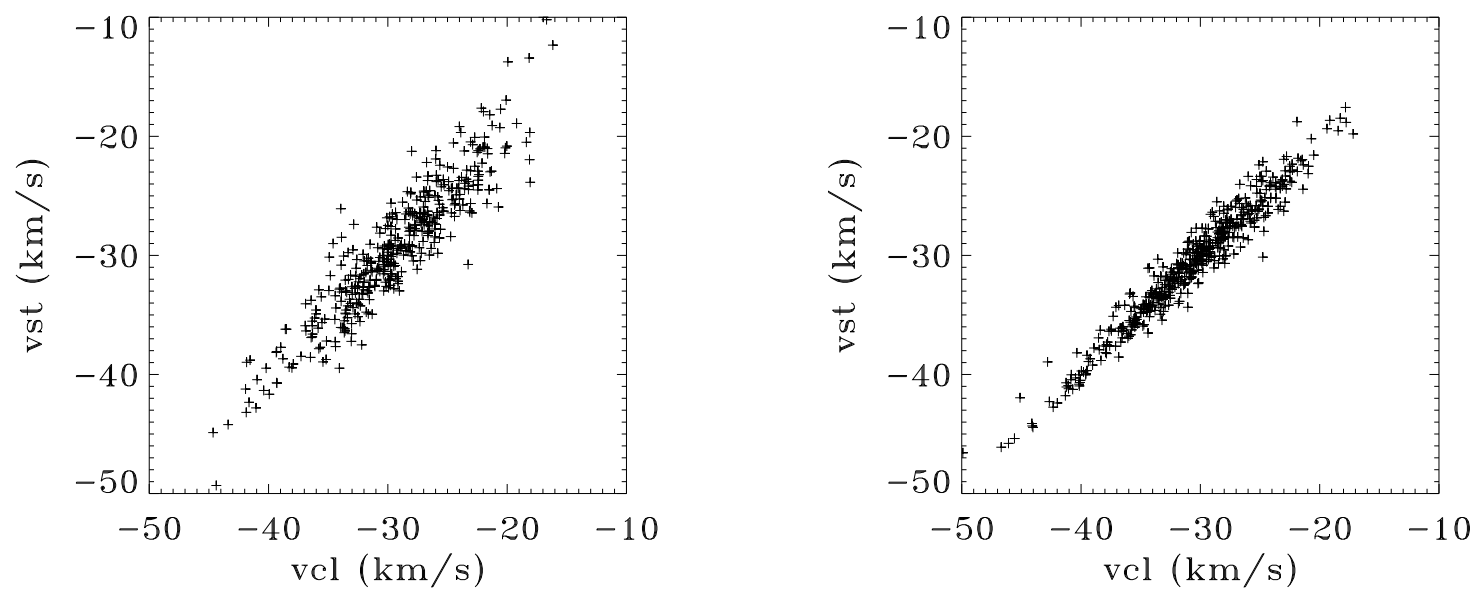

Fig. 3.- Comparison of slitless vs. classical FOCAS radial velocities of NGC 7293. The left and right figures correspond to chips 1 and 2, respectively. The PN's systemic radial velocity is $-27 \mathrm{~km} \mathrm{~s}^{-1}$, according to Meaburn et al. (2005). 


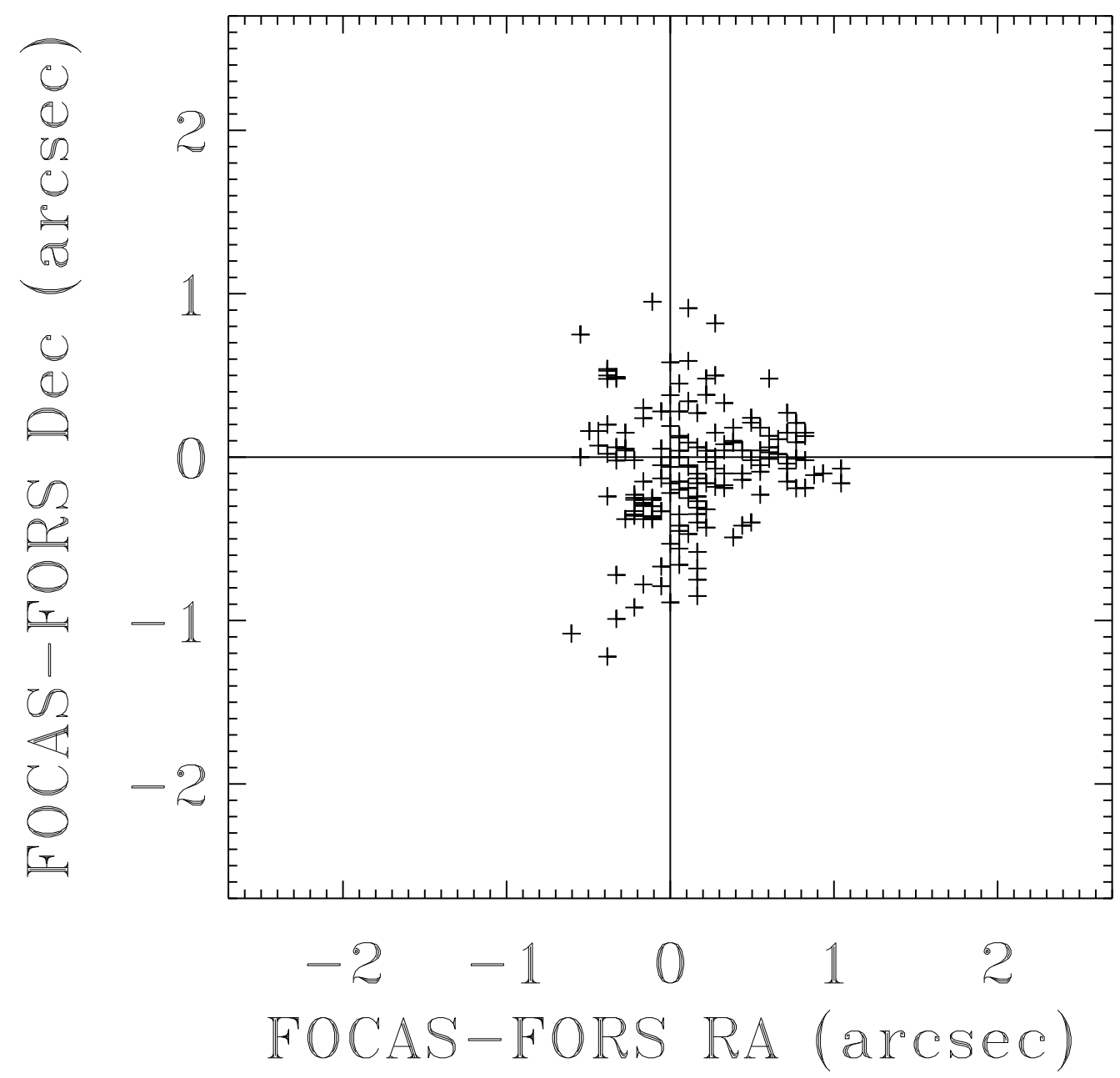

Fig. 4.- Comparison of equatorial coordinates for 162 PNs observed with both FORS and FOCAS. 


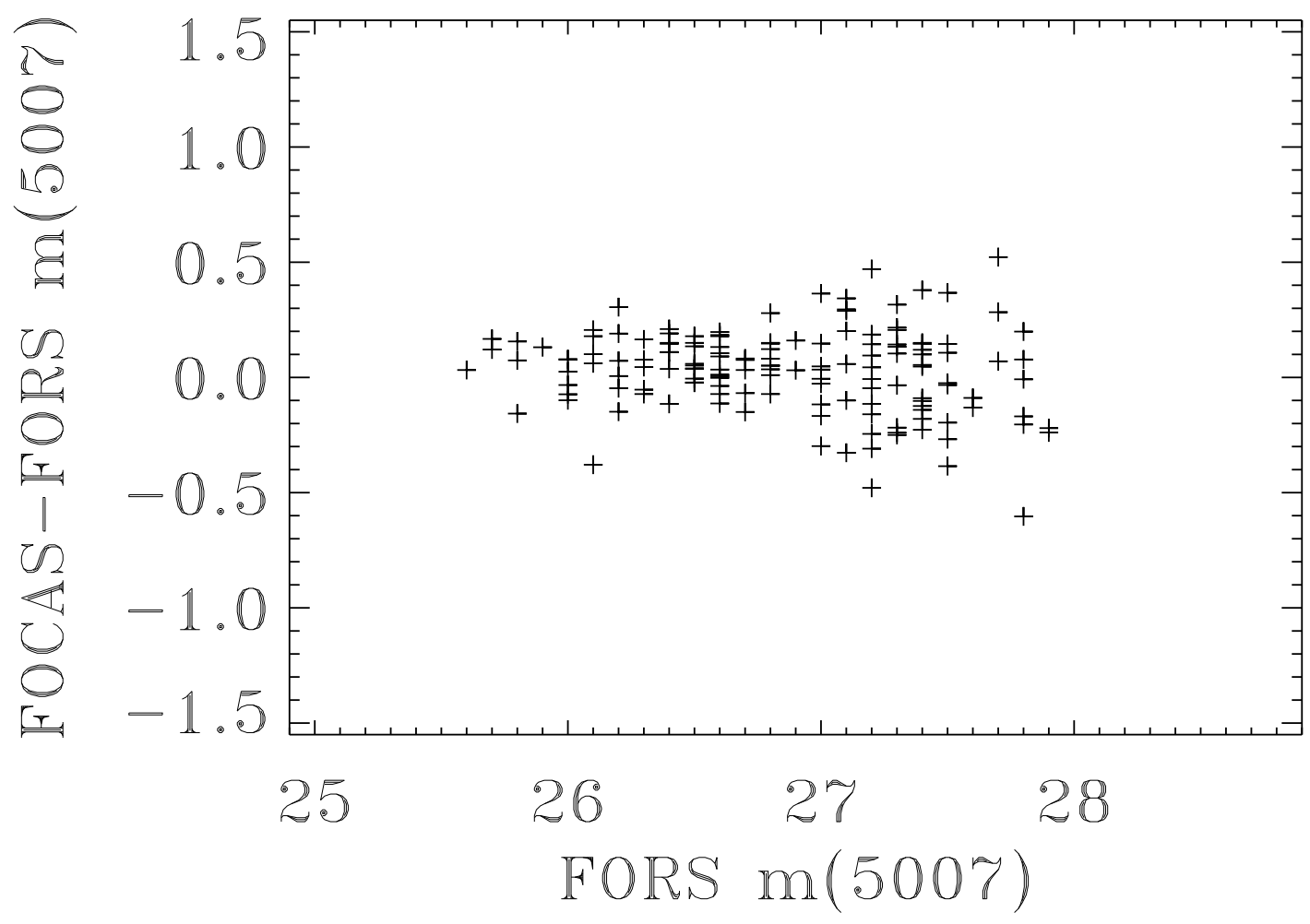

Fig. 5.- Differences (FOCAS - FORS) of Jacoby magnitudes $m(5007)$ plotted as a function of the FORS $m(5007)$.
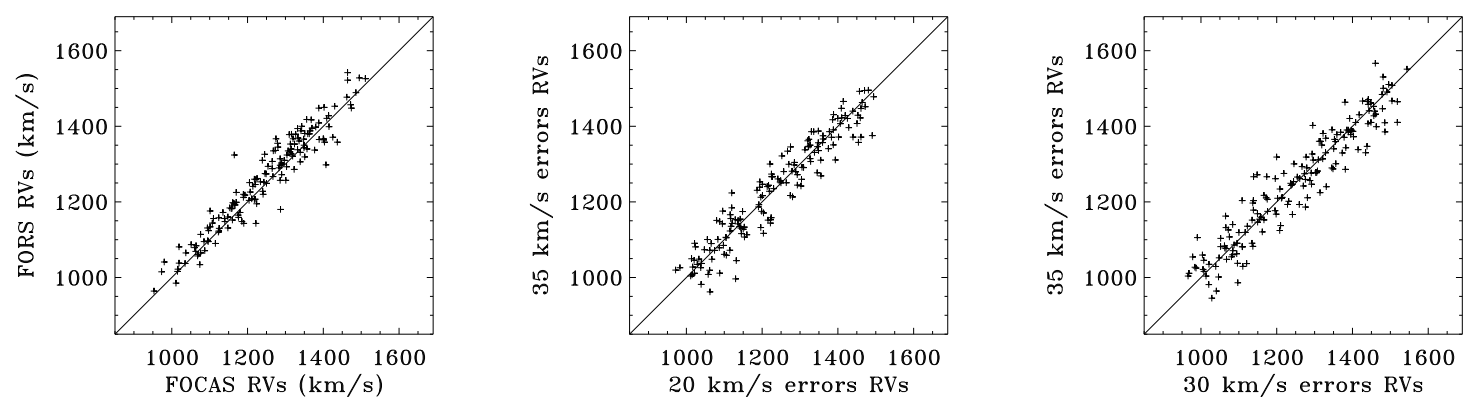

Fig. 6. - Left: comparison of heliocentric radial velocities for 158 PNs observed with both FORS and FOCAS. Center: random number simulations assuming errors of $35 \mathrm{~km} \mathrm{~s}^{-1}$ for FORS, and $20 \mathrm{~km} \mathrm{~s}^{-1}$ for FOCAS. Right: random number simulations assuming $30 \mathrm{~km} \mathrm{~s}^{-1}$ for FOCAS. We confirm that the FOCAS errors are not larger than $20 \mathrm{~km} \mathrm{~s}^{-1}$. 


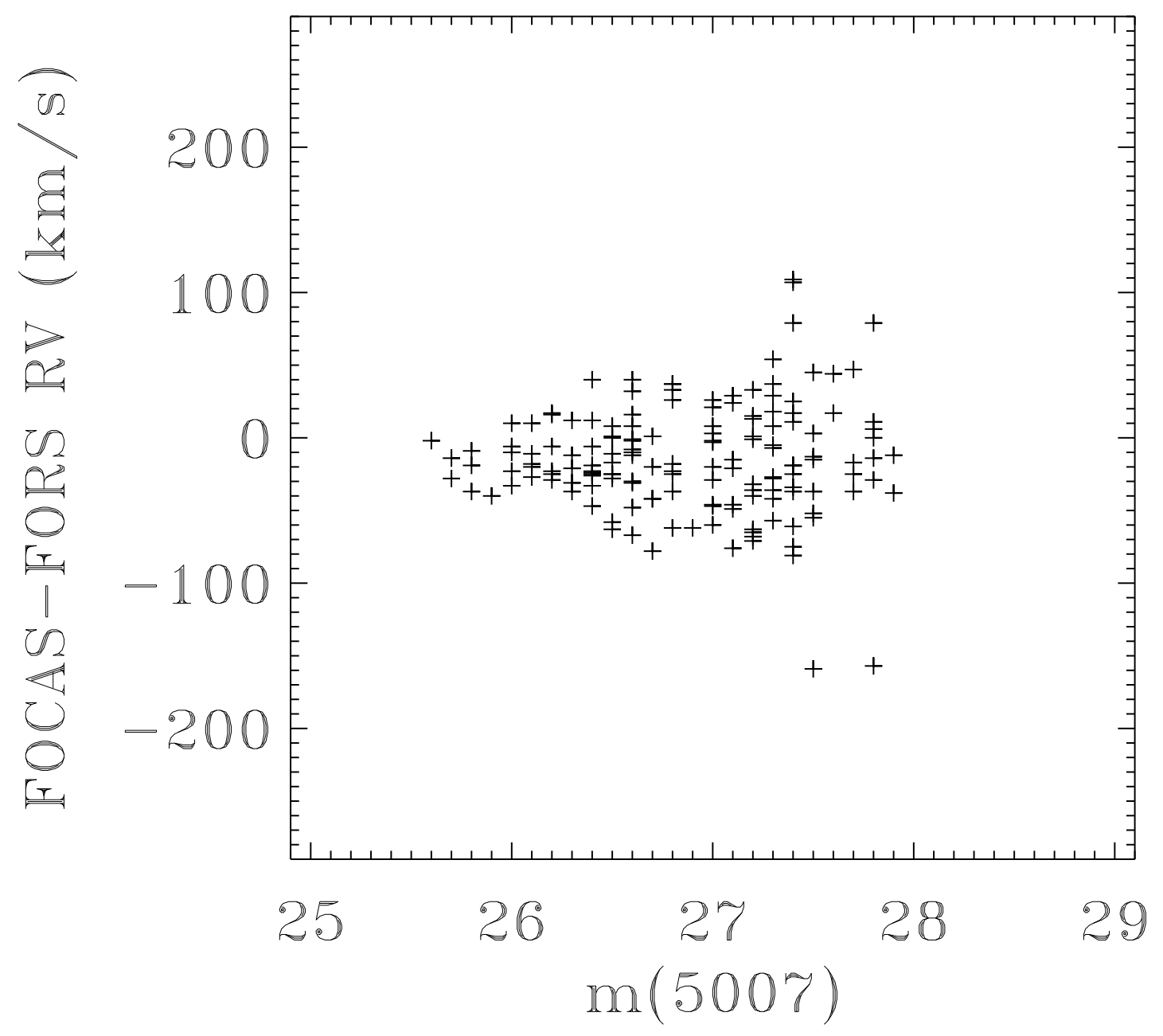

Fig. 7.- Velocity differences (FOCAS - FORS) plotted as a function of $m(5007)$. 

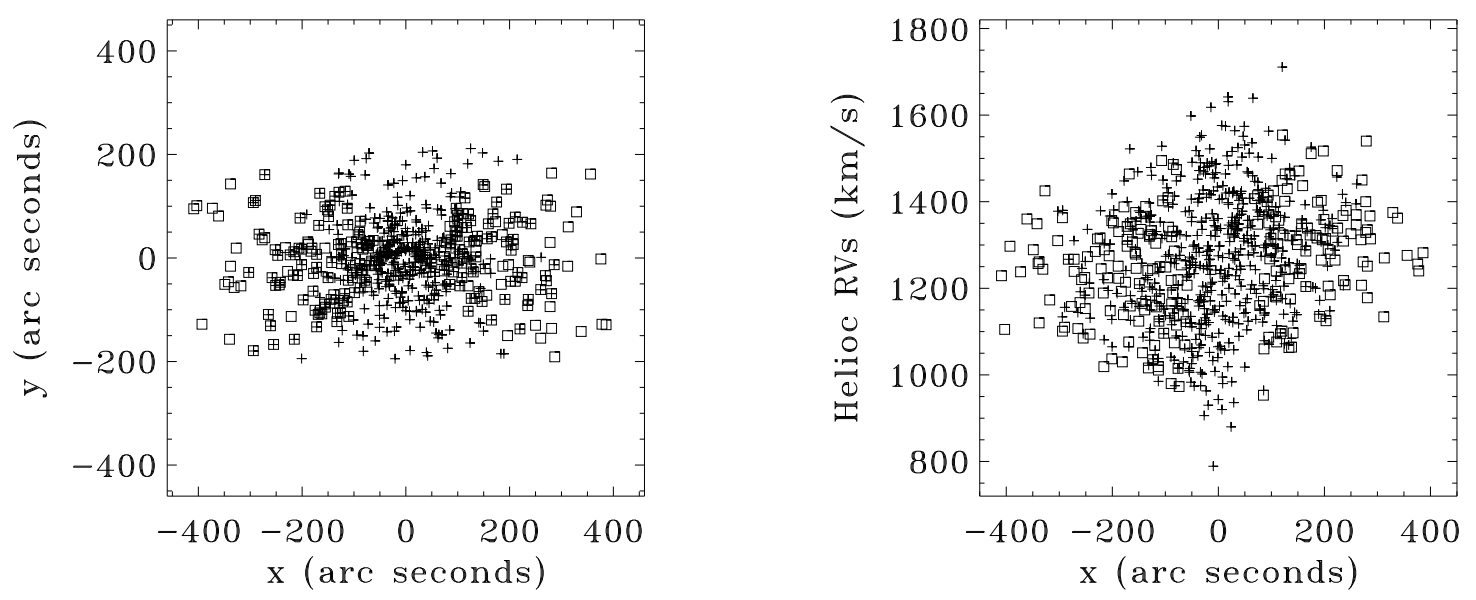

Fig. 8.- FORS (plus signs) and FOCAS (squares) PN detections. Left: positions of PNs relative to the center of light of NGC 4697, in arcsec. Right: radial velocities as a function of the $x$ coordinates. 


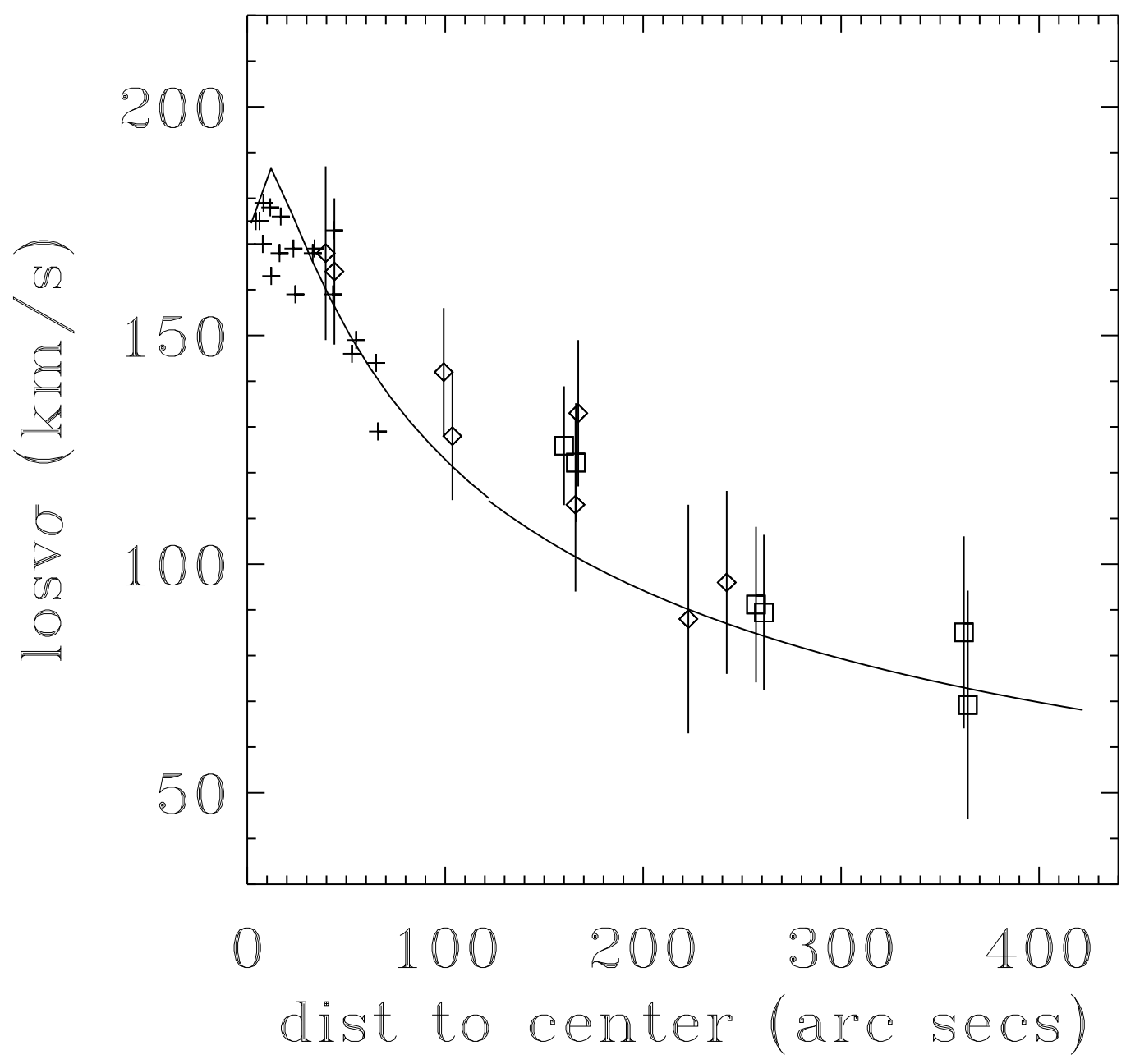

Fig. 9.- Line-of-sight velocity dispersion plotted as a function of average angular distance from the center of NGC 4697. Plus signs are measurements by Binney et al. (1990) on longslit, integrated-light spectra along the major axis. Diamonds are PN results from Paper I. Squares are PN results from FOCAS radial velocities. The solid line is the Hernquist (1990) analytical model with a constant $M / L$ ratio, adopting $R_{\mathrm{e}}=66$ arcsec, and a total mass of $1.5 \times 10^{11} M_{\odot}$. This is equivalent to $(M / L)_{B}=9$. 


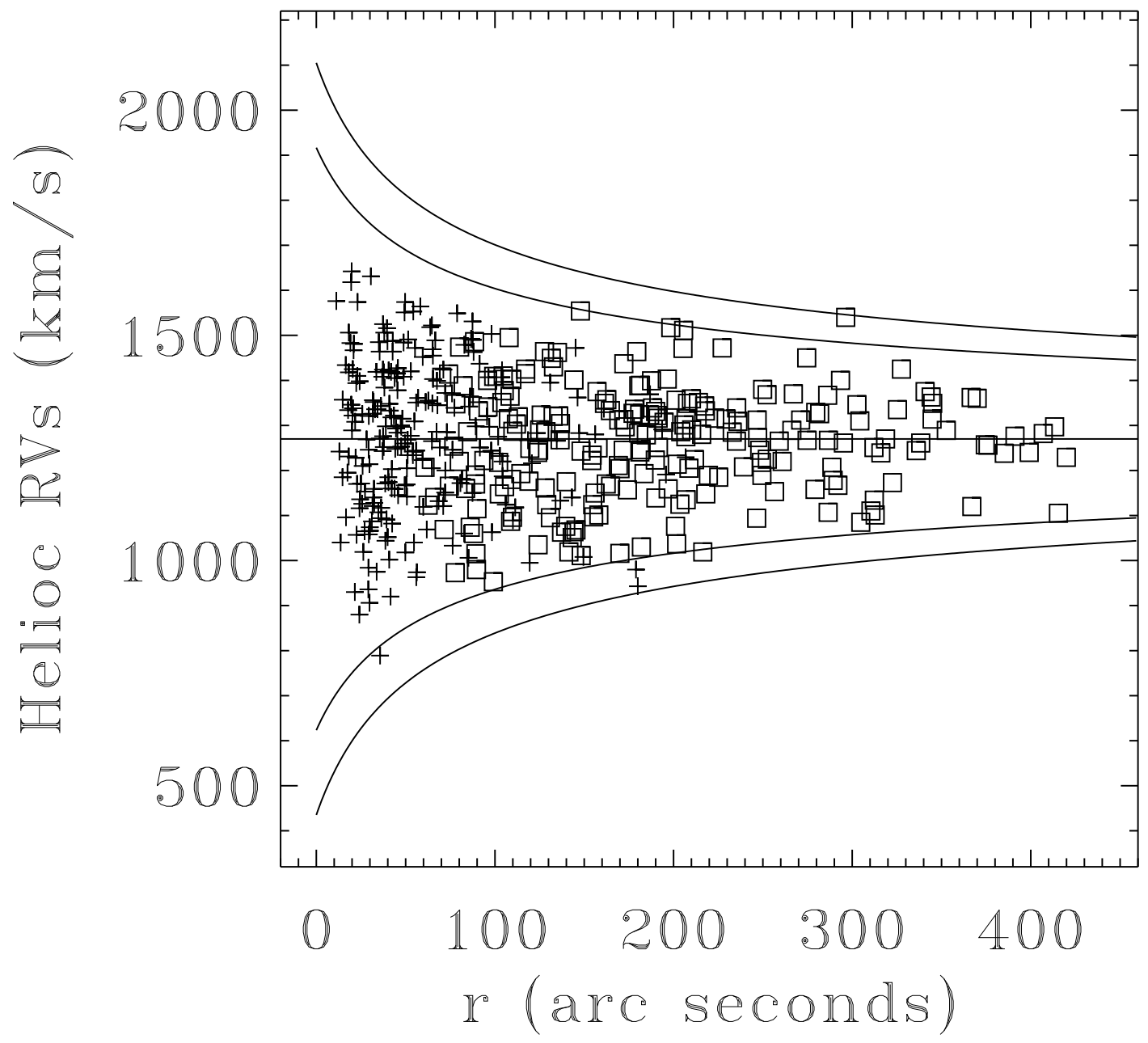

Fig. 10.- Individual FOCAS (squares) and FORS (plus signs) PN radial velocities plotted as a function of angular distance from the center of NGC 4697. We have only used FORS velocities of PNs from regions near the center, where we do not have FOCAS velocities. The solid lines are escape velocities for Hernquist models with $(M / L)_{B}=9$ (outer) and 5 (inner). 

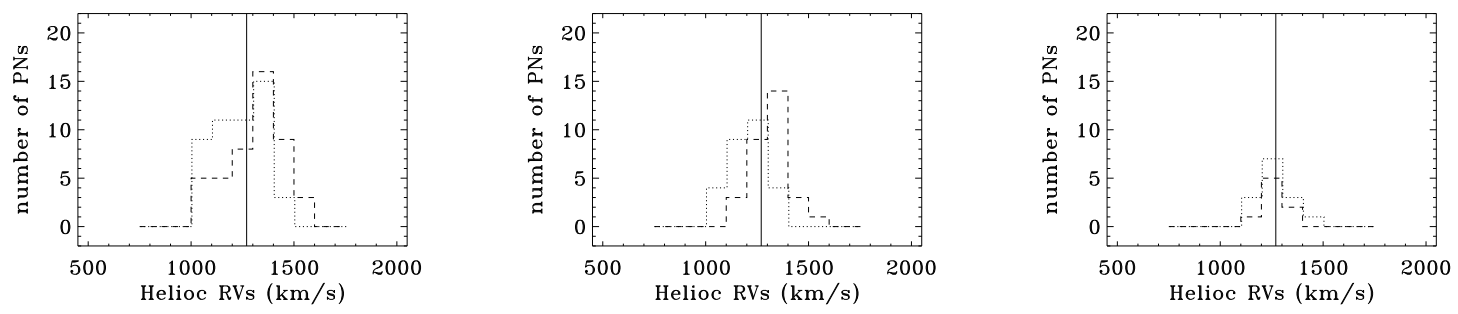

Fig. 11. - Left panel: radial velocity (FOCAS) histograms for PNs with $-200<x<-100$ (dotted line) and $100<x<200$ (dashed line). The vertical line indicates the systemic velocity, $1270 \mathrm{~km} \mathrm{~s}^{-1}$, taken from Paper I. There is an excess of positive relative velocities for negative $x$, but still the rotational signal is clear: at positive $x$ the PNs are preferentially moving away from us (in the same sense as the stars), while the opposite happens at negative $x$. Central panel: radial velocity (FOCAS) histograms for PNs with $-300<x<-200$ (dotted line) and $200<x<300$ (dashed line). The vertical line has always the same meaning. As we move outward, there is still a clear rotational signal (compare with the next panel). Right panel: radial velocity (FOCAS) histograms for PNs with $x<-300$ (dotted line) and $300<x$ (dashed line). No rotation is apparent here. 


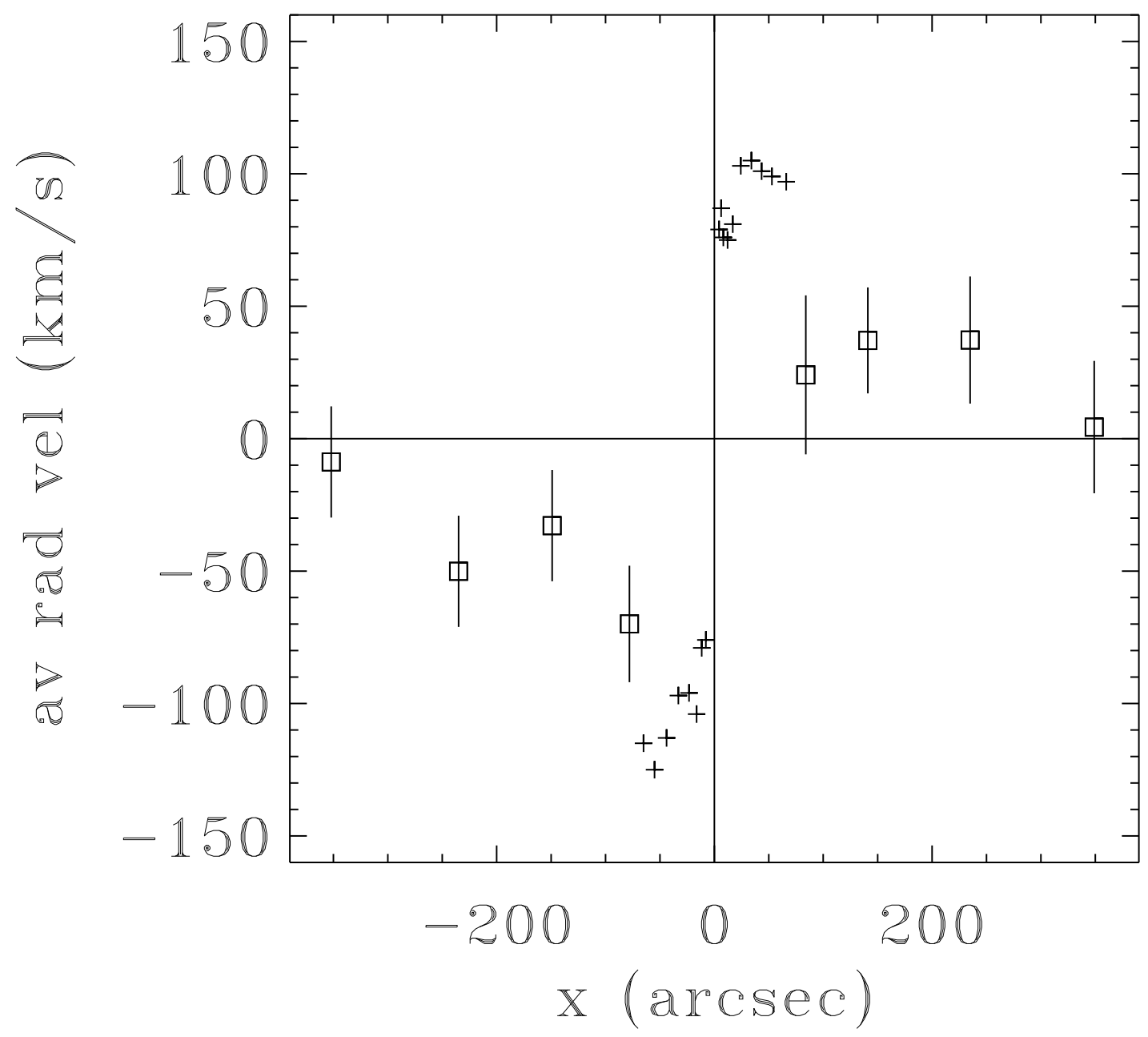

Fig. 12. - Average FOCAS PN radial velocity (squares) plotted as a function of average $x$ coordinate for eight PN groups, described in the text. Plus signs are velocities measured on long-slit, integrated-light spectra along the major-axis by Binney et al. (1990). The origin of velocities corresponds to the systemic velocity of NGC 4697, $1270 \mathrm{~km} \mathrm{~s}^{-1}$. The effective radius of NGC 4697 is 66 arcsec. 